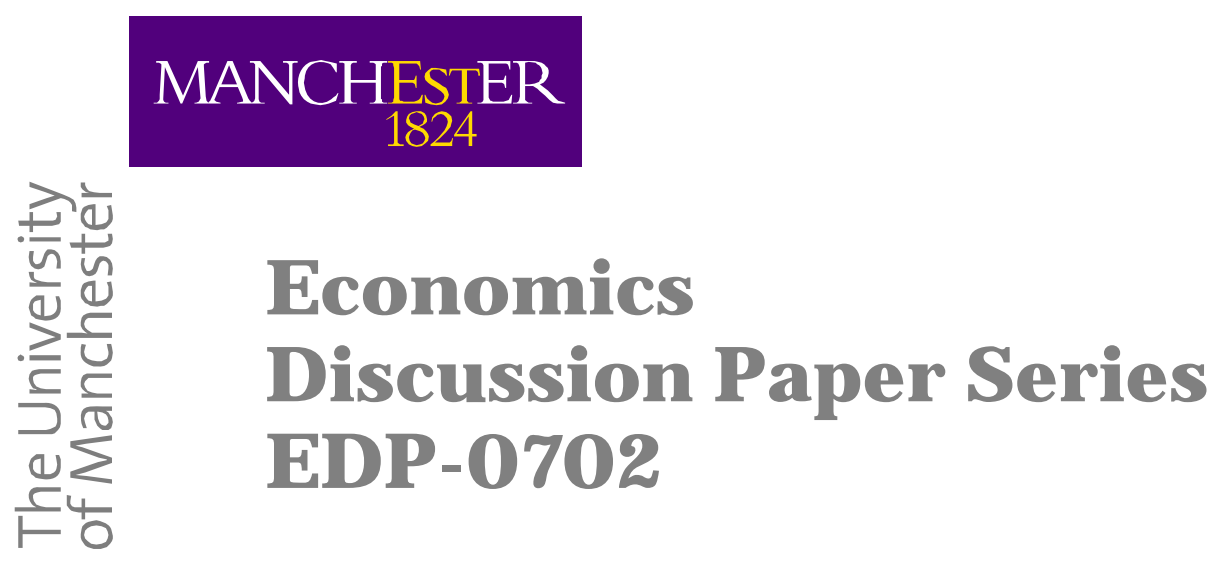

\title{
Existence and uniqueness of Nash equilibrium in electoral competition games: The hybrid case
}

Alejandro Saporiti

January 2007

Revised: December 2007

Economics

School of Social Sciences

The University of Manchester

Manchester M13 9PL 


\title{
Existence and uniqueness of Nash equilibrium in electoral competition games: The hybrid case*
}

\author{
Alejandro Saporiti ${ }^{\dagger}$
}

December $2007^{\ddagger}$

\begin{abstract}
This paper analyzes the traditional unidimensional, two-party electoral competition game when parties have mixed motivations, in the sense that they are interested in winning the election, but also in the policy implemented after the contest. In spite of having discontinuous payoffs, this game, refer to as the hybrid election game, is shown to be payoff secure and reciprocally upper semi-continuous. Conditional payoffs, however, are not quasi-concave. Hence, the existence of a pure strategy Nash equilibrium (PSNE) is ensured only if parties have homogenous interests in power. In that case, an equilibrium not only exists, but it is also unique. Instead, if parties have heterogeneous motivations, depending upon the relationship between the electoral uncertainty, the aggregate opportunism and its distribution across parties, a PSNE may or may not exist. The mixed extension, however, is always better reply secure. Therefore, a mixed strategy Nash equilibrium do indeed exist. These results generalize previous existence results in unidimensional electoral competition.
\end{abstract}

JEL Code: C72, D72, D78.

Keywords: Electoral competition, mixed motivations, discontinuous games, Nash equilibrium.

\section{Introduction}

Electoral competition between political parties is probably one of the most common and important features of every representative democracy. A large body of literature has been concerned with this process. ${ }^{1}$ This paper contributes to that literature by analyzing existence and uniqueness of Nash equilibrium in the traditional unidimensional, two-party political competition game when parties have mixed motivations, in the sense that they are interested in winning the election, but also in the policy implemented after the contest.

${ }^{*}$ I warmly thank the associate editor, two anonymous referees, Vincent Anesi, John Duggan, Jon Eguia, Paola Manzini, Marco Mariotti, Horst Zank and seminar participants at the Universities of Exeter, Manchester, Queen Mary (London), and Rochester, and at the meetings of the Association for Public Economic Theory (Marseille, 2005) and the Society for Social Choice and Welfare (Istanbul, 2006) for useful comments. Any remaining error is my own responsibility.

${ }^{\dagger}$ Economics, University of Manchester, Arthur Lewis Building, Oxford Road, Manchester M13 9PL, United Kingdom. E-mail: alejandro.saporiti@manchester.ac.uk.

${ }^{\ddagger}$ First Draft: April 2005.

${ }^{1}$ See, for instance, Roemer [27], Duggan [10] and Austen-Smith and Banks [3]. 
Formal models of electoral competition originated in the famous location model of Hotelling [17]. In the simplest version of this model, two political parties or candidates simultaneously announce a platform before the election, voters cast their votes, and the winner implements its announced policy. The basic idea captured by this model is that, in a unidimensional (left-right) political scale, each party can increase the number of votes by moving closer to the other party's position.

Thus, if parties care only about the outcome of the election, which is the Downsian hypothesis, and voters' preferences admit the existence of a Condorcet winner, as it is the case when they satisfy single-peakedness or single-crossing, then there is a unique Nash equilibrium in which both parties propose the same policy (Hotelling [17], Downs [8]). The equilibrium platform is of course the median of the distribution of voters' most preferred policies (Hinich [15]). This result also holds if there is uncertainty about individual preferences, provided that parties share a common prior about the location of the median ideal point. ${ }^{2}$ However, in that case platforms converge to the expected median (Calvert [6]).

Instead, if each party has an exogenous ideology, and cares only about how close the winning policy is relative to its own ideological position, which is the Wittman's [30] hypothesis, the convergence to the median ideal policy holds only if there is no uncertainty about voters' preferences (Calvert [6], Roemer [25], Duggan and Fey [12]). Otherwise, under uncertainty, the game has a pure strategy Nash equilibrium (PSNE), but equilibrium platforms do not converge (Roemer [26], $[27]) .{ }^{3}$ The reason for this is simple. As one party moves closer to the other, it becomes worse off in the event that it wins, but at the same time it increases its chances of winning the contest. Hence, parties face a trade-off between their ideology and winning the election, which results in an equilibrium where their positions are different.

Surprisingly, the existence of Nash equilibria in the hybrid case, where parties have preferences over policies, but also on the office itself, has not received much attention in the literature. This assumption, referred to as the mixed motivation assumption (MMA), is certainly more realistic than the previous hypotheses about parties' motivations. For example, the MMA naturally arises if professional politicians are parties' leaders. ${ }^{4}$ Since politicians may namely be interested in their career and, therefore, in winning the election, while regular party members may care more about policy outcomes, it is natural to expect that both objectives will enter into the party payoff function with some weight. ${ }^{5}$ Of course, these weights need not be the same across parties. Hence, heterogeneous interests arise quite naturally as well.

To the best of my knowledge, Calvert [6] was the first to show that, if parties have mixed motivations and there is no uncertainty about the median ideal point, then there exists a unique PSNE in which platforms converge to the median position, provided that parties' ideal policies are not on the same side of the median. Otherwise, the ideal point that is closest to the median is a Nash equilibrium.

Although Calvert [6] did not address the issue of equilibrium existence under uncertainty, he showed that, if motivations are homogenous across parties, in the sense that they assign the same relative weight on holding office, "small departures" from the office motivation and certainty model

\footnotetext{
${ }^{2}$ See Bernhardt et al. [5] for a model with private pooling, where candidates receive private signals about voters' preferences before committing to their policy platforms.

${ }^{3}$ Morton [22] provided experimental evidence which supports the fact that uncertainty over voters' preferences is a major determinant of platform divergence when candidates are ideological.

${ }^{4}$ See Ortuño Ortín [23] for a model in this line.

${ }^{5}$ Using experimental evidence about the probabilistic ideological election game, Morton [22] found that subjects in the laboratory placed a weight of approximately $68 \%$ on the expected payoffs from policies and $32 \%$ on winning.
} 
lead to "small changes" in the results. Thus, the conclusion that is possible to draw from his analysis is that in the limit, when the game is arbitrarily close to the Downsian game, (represented in Figure 1 below in the southwest corner), a PSNE always exists.



Information about voters' preferences

Figure 1: Electoral competition with homogeneous motives

But, what about equilibrium existence in other cases? As Figure 1 illustrates, even if we maintain the assumption that parties' motives are homogeneous, there are still regions in the two-dimensional "Motivation-Information Box" (shown as the dotted line) where the existence of electoral equilibria remains an open question. Needless to say that this is also the case if we add a third dimension into the analysis, differentiating party 1's motivations from those of party 2 .

More importantly, it is worth noting that answering such questions is by no means a trivial exercise, because the hybrid election game is an infinite action game with discontinuous payoffs. In effect, it is well known in the literature on political competition under uncertainty that the probability of winning the election is discontinuous on the diagonal. This in turns may preclude the existence of best replies and, therefore, the best reply correspondence need not be nonempty valued, let alone upper hemi-continuous.

Such a problem does not affect the probabilistic Downsian electoral game (southeast corner of the box), which always has a unique Nash equilibrium in pure strategies. Moreover, it does not affect the probabilistic Wittman election game (northeast corner) either. As Roemer [26] showed, purely ideological parties have continuous payoffs, in spite of the discontinuities of the probability of winning function, so that best reply correspondences are always well defined. However, it could be a problem for the hybrid game.

The first attempt to tackle this problem and find out the implications of the MMA for the existence of Nash equilibria in electoral games with uncertainty was done by Ball [4]. 6 This paper was the first to correctly point out that the hybrid game may fail to possess a Nash equilibrium in pure strategies. Moreover, it provided sufficient conditions for the existence of mixed strategy

\footnotetext{
${ }^{6}$ Before that, four papers studied candidates' behavior with mixed motives. However, the first one, due to Mitchell [21], has the disadvantage that the ideological component of the payoff function is given by the cost of departing from the party's ideological position, instead of by the expected utility over candidates' proposals. The next two, due to Wittman [30] and [31], wrongly assumed continuity of the probability of winning the election and quasi-concavity of conditional payoffs, respectively, which are usually violated in the hybrid game. And, finally, Morton [22] analyzed perfect Bayesian equilibria in a game where the type of each party is private information.
} 
Nash equilibria (MSNE). It has the disadvantage, however, that the probability of winning function is exogenously given. That is, it is taken as a primitive, instead of being derived endogenously from parties' uncertainty about the median ideal point. Furthermore, the paper is also silent about when a PSNE may exist, and how equilibria (either in pure or mixed strategies) may look like. It doesn't analyze uniqueness either.

In this paper, we reconsider the problem of existence and uniqueness of Nash equilibrium in the hybrid election game with stochastic preferences. First, we show that, under the MmA, parties' payoffs are neither continuous nor semi-continuous. This implies that, unless both have homogenous interests in power, the less ideological party may have incentives to undercut the other party's position, moving its own position to an alternative arbitrarily close to the platform of the other. In cases like that, best reply correspondences are empty and, therefore, a PSNE does not exist. In particular, this happens if one party is office motivated and the other entirely ideological.

Second, we prove that, in spite of having discontinuous payoffs, the hybrid game is payoff secure and reciprocally upper semi-continuous. So, following Reny [24], we conclude that the blame for the nonexistence of a Nash equilibrium in pure strategies for all parametric conditions can be fully assigned to the violation of quasi-concavity. Moreover, we show that non-convexities matter only if the relative interest in holding office is heterogeneous across parties. ${ }^{7}$ By contrast, in the homogeneous case, regardless of whether the motivations are mixed or not, we prove that a unique pure strategy Nash equilibrium always exists, obtaining as a particular case of that result the well known Downsian and Wittman electoral equilibria.

Finally, in the case in which parties are distinctly concerned about winning the election, we argue that the relatively more ideological party must take into account that the rival may be willing to "copy" its platform. To avoid this, it must draw its proposal from a probability distribution. This induces a similar behavior in the opposition. The paper proves that a pair of probability measures that are best responses to each other always exists. ${ }^{8}$ Specifically, it shows that the mixed extension of the hybrid election game is better reply secure. Therefore, invoking Reny [24], we conclude that a mixed strategy Nash equilibrium do indeed exist. ${ }^{9}$ This extends Ball [4] to the case where the function mapping candidates' platforms to their probabilities of winning the election is derived from primitives about electoral uncertainty, and it generalizes previous existence results in unidimensional electoral competition.

\section{The model}

Consider a society with two political parties (candidates), noted 1 and 2 , and a continuum of voters, indexed by a type $\theta \in[0,1]$. Assume $\theta$ is distributed according with a continuous distribution function $F$, with density $f$, and $F\left(\theta_{m}\right)=1 / 2$ for some $\theta_{m} \in(0,1)$. Let $[0,1]$ denote the policy space. Following Duggan and Fey [12], Bernhardt et al. [5] and many others in the literature, suppose individual preferences are continuous, single-peaked and symmetric on [0,1], and adopt the usual utility representation given in A1:

Assumption (A1) $u(x ; \theta)=-|x-\theta|$, where $|\cdot|$ is the absolute value on $\mathbb{R}$.

\footnotetext{
${ }^{7}$ The necessity of this feature for nonexistence of PSNE was not addressed before, and it considerably sharpens Ball's [4] results. I thank the referee for this remark.

${ }^{8}$ Saporiti [28] offers more details about how equilibria may look like in a tractable version of the model.

${ }^{9}$ For other applications of mixed strategies in electoral competition, see Kramer [18], Laslier [20], Aragones and Palfrey [2], Duggan and Jackson [11] and Bernhardt et al. [5].
} 
Let $A_{i}=[0,1]$ denote party $i$ 's strategy set, with generic action (platform) $x_{i}$, and $A=A_{1} \times A_{2}$ the set of all strategy profiles. We refer to $D(A)=\left\{\left(x_{1}, x_{2}\right) \in A: x_{1}=x_{2}\right\}$ as the diagonal of the product of the strategy sets; or, for conciseness, just as "the diagonal". Like in the ordinary electoral competition game, assume each party simultaneously announces a platform $x_{i}$ in $[0,1]$. Then, given a proposal $\left(x_{1}, x_{2}\right) \in A$, each voter votes for the policy he likes the most, where $S\left(x_{1}, x_{2}\right)=\left\{\theta \in[0,1]: u\left(x_{1} ; \theta\right)>u\left(x_{2} ; \theta\right)\right\}$ denotes the set of types that support $x_{1}$. The party whose platform obtains more than half of the votes wins the election, and its policy is implemented. Ties are broken by a random draw, so that each party wins with probability one half if all voters are indifferent between the two platforms.

In addition to the uncertainty due to the possibility of a tie, parties are also uncertain about voters' preferences. Following Roemer [27], we assume parties perceive the fraction of types that supports their platform with a certain noise. Formally, let $\xi$ be an error term, distributed according with a continuous distribution function $H$ on $[-\beta, \beta], \beta>0$, with density $h$, and $H(0)=1 / 2$.

Assumption (A2) For each pair $\left(x_{1}, x_{2}\right) \notin D(A)$, parties believe that the fraction of types supporting $x_{1}$ is $F\left(S\left(x_{1}, x_{2}\right)\right)+\xi$, and $1 / 2$ if the strategy profile $\left(x_{1}, x_{2}\right) \in D(A) .{ }^{10}$

Thus, given $\left(x_{1}, x_{2}\right) \in A$, the probability that party 1 attaches to winning the election is

$$
p\left(x_{1}, x_{2}\right)= \begin{cases}1-H\left(1 / 2-F\left(S\left(x_{1}, x_{2}\right)\right)\right) & \text { if }\left(x_{1}, x_{2}\right) \notin D(A) \\ 1 / 2 & \text { if }\left(x_{1}, x_{2}\right) \in D(A)\end{cases}
$$

where it is understood that $H(y)=0$ if $y \leq-\beta$ and $H(y)=1$ if $y \geq \beta$. The probability that party 2 attaches to winning the contest takes an analogous form, and is given by $1-p\left(x_{1}, x_{2}\right)$.

It is immediate from (1) that $p\left(x_{1}, x_{2}\right)$ is discontinuous at every point on $D(A)$, except at $\left(\theta_{m}, \theta_{m}\right)$, where $\theta_{m}$ is the median ideal point. As it happens in other applications, notably in price competition, these discontinuities are created by the tie-breaking rule implicit in (1), according to which, if parties coincide in their platforms at a certain point on the diagonal, indifferent voters split equally among them, regardless of their distribution in a neighboring point arbitrarily close to that location.

This "equally sharing" rule is the standard tie-breaking rule used in the literature on electoral competition under uncertainty. And, except for a very recent article by Duggan and Jackson [11] on multidimensional electoral competition, which assumes indifferent voters play symmetric voting strategies, I am aware of no systematic analysis of political competition with endogenous sharing rules or other tie-breaking rules. ${ }^{11}$ This is particularly true in the unidimensional twoparty election game since, with the exception of the hybrid game analyzed here, and Bernhardt et al.'s [5] probabilistic Downsian game with private pooling, the rest always admit under reasonable conditions the existence of a PSNE.

Let $\chi_{i} \geq 0$ be the intrinsic value that party $i$ places on being in office, and $\theta^{i}$ the preferences on $[0,1]$ that it represents. Define the function $\psi(x, y ; \theta)=u(x ; \theta)-u(y ; \theta)$. Parties' objective functions are defined as follows:

Assumption (A3) For each strategy profile $\left(x_{1}, x_{2}\right) \in A$, party 1's payoff function is given by $\pi_{1}\left(x_{1}, x_{2}\right)=p\left(x_{1}, x_{2}\right)\left(\psi\left(x_{1}, x_{2} ; \theta^{1}\right)+\chi_{1}\right)$. Similarly, party 2's payoff is $\pi_{2}\left(x_{1}, x_{2}\right)=(1-$ $\left.p\left(x_{1}, x_{2}\right)\right)\left(\psi\left(x_{2}, x_{1} ; \theta^{2}\right)+\chi_{2}\right)$.

\footnotetext{
${ }^{10}$ Recall that, by A1, for each $\left(x_{1}, x_{2}\right) \notin D(A), F\left(I\left(x_{1}, x_{2}\right)\right)=0$, where $I\left(x_{1}, x_{2}\right)=\left\{\theta: u\left(x_{1} ; \theta\right)=u\left(x_{2} ; \theta\right)\right\}$.

${ }^{11}$ In price competition, this issue has been recently addressed by Hoernig [16].
} 
In words, A3 says that in our model political parties have preferences over the implemented policy, but also on the office itself. Moreover, the payoffs are separable in these arguments. Of course, this need not be the case, and one can imagine situations where parties care more about policy if they win the election than if they lose. In addition, there might be other motivations to consider. For example, a party may have preferences over its margin of victory (vote share), apart from policies and winning the election. However, A3 seems the simplest case to begin with.

It is easy to see that the payoff functions of the traditional models of electoral competition are particular instances of A3. In effect, if $\chi_{i}=0$, then $\pi_{i}(x)$ represents the objective function of the Wittman game, where parties seek the best policy outcome from the election. On the other hand, if $\chi_{i} \rightarrow+\infty$, then each party maximizes the probability of winning the contest, without caring at all about policies, which is the classical assumption of the Downsian game. ${ }^{12}$ The hybrid case, where $\forall i, \chi_{i}>0$, and $\chi_{1}$ is not necessarily equal to $\chi_{2}$, is somewhere between these two extreme and symmetric cases.

Regarding the specification of the MMA adopted in A3, note that we follow Wittman [30], Ball [4], Duggan [9] and Austen-Smith and Banks [3]; and, more generally, Ferejohn's [13] idea that policymakers enjoy some rents from being in power. But there is an alternative way to express the mixed motivations. Following Calvert [6], Aragones and Palfrey [1] and Groseclose [14] assume that the objective function of each party is a linear combination of the probability of winning the election and a second component corresponding to its policy preferences. Specifically, using $\hat{\pi}_{j}$ to denote this alternative formulation of parties' payoffs, we have that

$$
\begin{aligned}
& \hat{\pi}_{1}\left(x_{1}, x_{2}\right)=\lambda_{1} p\left(x_{1}, x_{2}\right)+\left(1-\lambda_{1}\right) p\left(x_{1}, x_{2}\right) \psi\left(x_{1}, x_{2} ; \theta^{1}\right) \\
& \hat{\pi}_{2}\left(x_{1}, x_{2}\right)=\lambda_{2}\left(1-p\left(x_{1}, x_{2}\right)\right)+\left(1-\lambda_{2}\right)\left(1-p\left(x_{1}, x_{2}\right)\right) \psi\left(x_{2}, x_{1} ; \theta^{2}\right),
\end{aligned}
$$

where $\lambda_{j}$ is the weight that party $j$ assigns on holding office. Simple algebraic manipulation shows that, for the main purpose of our analysis, these two formulations are equivalent. In effect, denoting $\chi_{j}=\frac{\lambda_{j}}{1-\lambda_{j}}$, it follows that

$$
\begin{aligned}
\hat{\pi}_{1}\left(x_{1}, x_{2}\right) & =\left(1-\lambda_{1}\right) p\left(x_{1}, x_{2}\right)\left(\psi\left(x_{1}, x_{2} ; \theta^{1}\right)+\chi_{1}\right), \\
& =\left(1-\lambda_{1}\right) \pi_{1}\left(x_{1}, x_{2}\right), \\
\hat{\pi}_{2}\left(x_{1}, x_{2}\right) & =\left(1-\lambda_{2}\right)\left(1-p\left(x_{1}, x_{2}\right)\right)\left(\psi\left(x_{2}, x_{1} ; \theta^{2}\right)+\chi_{2}\right), \\
& =\left(1-\lambda_{2}\right) \pi_{2}\left(x_{1}, x_{2}\right) .
\end{aligned}
$$

Thus, since $\lambda_{j} \in(0,1)$, the equations above show that, for all $j=1,2, \hat{\pi}_{j}$ is a strictly increasing linear transformation of $\pi_{j}$. Hence, the results about equilibrium existence and uniqueness derived in Sections 3 and 4, for pure and mixed strategies, respectively, extend directly to this alternative way to express the MMA.

Finally, with respect to the technical consequences of A3, note that together with (1) it implies that parties' payoffs are discontinuous on $D(A)$, except at $\left(\theta_{m}, \theta_{m}\right)$. Furthermore, as Example 1 and Lemma 1 below show, $\pi_{i}$ is neither upper semi-continuous nor lower semi-continuous on $A,{ }^{13}$

\footnotetext{
${ }^{12}$ Notice that $\tilde{\pi}_{i}(x)=1 / \chi_{i} \pi_{i}(x)$ represents the same preferences than $\pi_{i}(x)$, and that $\lim _{\chi_{1} \rightarrow \infty} \tilde{\pi}_{1}(x)=p(x)$ and $\lim _{\chi_{2} \rightarrow \infty} \tilde{\pi}_{2}(x)=1-p(x)$.

${ }^{13} \mathrm{~A}$ function $f: Y \rightarrow \mathbb{R}$ on a nonempty and compact subset $Y \subset \mathbb{R}^{k}$ is upper semi-continuous (u.s.c.) at $y$ if for any sequence $\left\{y^{n}\right\} \subseteq Y$ such that $y^{n} \rightarrow y, \lim _{\sup _{n \rightarrow \infty}} f\left(y^{n}\right) \leq f(y)$. On the other hand, $f$ is lower semi-continuous (l.s.c) at $y$ if $-f$ is u.s.c. at $y$.
} 
and except for the case where $\chi_{1}=\chi_{2}$, the sum of the payoffs $\Pi=\pi_{1}+\pi_{2}$ is also discontinuous on $D(A)$. The reason for this is the discontinuities in the probability of winning function entail discontinuous shifts of the electorate from one party's platform to the other. Therefore, if platforms are not equally profitable for parties, total payoff also changes discontinuously.

Example 1 Suppose $\xi$ and $\theta$ are uniformly distributed, and assume that $\theta^{1}<\theta_{m}<\theta^{2}$. Take a point $x_{\delta}=\left(\theta_{m}+\delta, \theta_{m}+\delta\right) \in D(A), \delta>0$, and a sequence $\left(x_{1}^{n}, x_{2}^{n}\right)=\left(\theta_{m}+\delta-\frac{1}{n}, \theta_{m}+\delta\right)$, that converges to $x_{\delta}$ from the left as $n \rightarrow \infty$. Since $x_{1}^{n}<x_{2}^{n}, \pi_{1}\left(x_{1}^{n}, x_{2}^{n}\right)=\left(\frac{\delta}{2 \beta}-\frac{1}{4 \beta n}+\frac{1}{2}\right)\left(\chi_{1}+\right.$ $\left.\frac{1}{n}\right)$. Then, $\lim _{n \rightarrow \infty} \pi_{1}\left(x_{1}^{n}, x_{2}^{n}\right)=\frac{\chi_{1}}{2}+\frac{\delta \chi_{1}}{2 \beta}>\frac{\chi_{1}}{2}=\pi_{1}\left(x_{\delta}\right)$. Similarly, consider now a sequence $\left(\hat{x}_{1}^{n}, \hat{x}_{2}^{n}\right)=\left(\theta_{m}+\delta+\frac{1}{n}, \theta_{m}+\delta\right)$ that converges to $x_{\delta}$ from the right. Since $\hat{x}_{1}^{n}>\hat{x}_{2}^{n}, \pi_{1}\left(\hat{x}_{1}^{n}, \hat{x}_{2}^{n}\right)=$ $\left(\frac{1}{2}-\frac{\delta}{2 \beta}-\frac{1}{4 \beta n}\right)\left(\chi_{1}-\frac{1}{n}\right)$. Then, $\lim _{n \rightarrow \infty} \pi_{1}\left(\hat{x}_{1}^{n}, \hat{x}_{2}^{n}\right)=\frac{\chi_{1}}{2}-\frac{\delta \chi_{1}}{2 \beta}<\frac{\chi_{1}}{2}=\pi_{1}\left(x_{\delta}\right)$. Hence, $\pi_{1}$ is neither l.s.c. nor u.s.c. at $x_{\delta}$.

Lemma $1 \Pi=\pi_{1}+\pi_{2}$ is continuous on $A$ if only if $\chi_{1}=\chi_{2}$.

Proof. By A3, $\Pi\left(x_{1}, x_{2}\right)=\phi\left(x_{1}, x_{2} ; \theta^{1}, \theta^{2}\right)+\psi\left(x_{2}, x_{1} ; \theta^{2}\right)+\chi_{2}$, where $\phi\left(x_{1}, x_{2} ; \theta^{1}, \theta^{2}\right)=$ $p\left(x_{1}, x_{2}\right)\left[\psi\left(x_{1}, x_{2} ; \theta^{1}\right)-\psi\left(x_{2}, x_{1} ; \theta^{2}\right)+\left(\chi_{1}-\chi_{2}\right)\right]$. Since $u\left(\cdot ; \theta^{2}\right)$ is continuous in $x$ and $\chi_{2}$ is a constant, $\psi\left(\cdot, \cdot ; \theta^{2}\right)+\chi_{2}$ is continuous on $A$. On the other hand, for any $\bar{x}_{1} \neq \bar{x}_{2}, p(\cdot)$ is continuous at $\bar{x}=\left(\bar{x}_{1}, \bar{x}_{2}\right)$, because $H$ and $F$ are continuous functions. Hence, by A1, $\phi\left(\cdot, \cdot ; \theta^{1}, \theta^{2}\right)$ is also continuous. On the contrary, if $\bar{x}_{1}=\bar{x}_{2}$, then $p\left(\bar{x}_{1}, \bar{x}_{2}\right)=1 / 2$. Consider a sequence $x^{n}=\left(x_{1}^{n}, x_{2}^{n}\right) \subseteq A$, such that $\left(x_{1}^{n}, x_{2}^{n}\right) \rightarrow\left(\bar{x}_{1}, \bar{x}_{2}\right)$. Note that $\phi\left(\bar{x}_{1}, \bar{x}_{2} ; \theta^{1}, \theta^{2}\right)=\left(\chi_{1}-\chi_{2}\right) / 2$. Thus, if $\chi_{1}=\chi_{2}$, $\phi\left(\bar{x}_{1}, \bar{x}_{2} ; \theta^{1}, \theta^{2}\right)=0$. Taking the limit of $\phi\left(x_{1}^{n}, x_{2}^{n} ; \theta^{1}, \theta^{2}\right)$ with $\left(x_{1}^{n}, x_{2}^{n}\right) \rightarrow\left(\bar{x}_{1}, \bar{x}_{2}\right)$, we have that $\lim _{x^{n} \rightarrow \bar{x}} \phi\left(x_{1}^{n}, x_{2}^{n} ; \theta^{1}, \theta^{2}\right)=\lim _{x^{n} \rightarrow \bar{x}} p\left(x_{1}^{n}, x_{2}^{n}\right)\left[\psi\left(x_{1}^{n}, x_{2}^{n} ; \theta^{1}\right)-\psi\left(x_{2}^{n}, x_{1}^{n} ; \theta^{2}\right)\right]=0$, since $p\left(x_{1}^{n}, x_{2}^{n}\right)$ is bounded and $\psi\left(x_{1}^{n}, x_{2}^{n} ; \theta^{1}\right)-\psi\left(x_{2}^{n}, x_{1}^{n} ; \theta^{2}\right)$ converges to zero as $x^{n} \rightarrow \bar{x}$. Therefore, $\Pi$ is continuous on $A$.

Instead, if $\chi_{1} \neq \chi_{2}$, then the term $p\left(x_{1}^{n}, x_{2}^{n}\right)\left(\chi_{1}-\chi_{2}\right)$ does not converge to $\left(\chi_{1}-\chi_{2}\right) / 2$ as $x^{n} \rightarrow \bar{x}$, except at $\left(\bar{x}_{1}, \bar{x}_{2}\right)=\left(\theta_{m}, \theta_{m}\right)$. Hence, the discontinuities of the probability of winning the election create discontinuities not only in each payoff, but also in the sum.

Note that Example 1 and Lemma 1 stand in sharp contrast with Roemer [26], where it is shown that, in spite of the discontinuities of the probability of winning function, purely ideological parties have continuous payoffs. More importantly, it suggests that even if only one party assigns an arbitrarily small weight on winning the election, this completely invalidates Roemer's [26] and [27] equilibrium analysis, leaving the question of existence without answer. This disturbing observation constitutes one of the main motivations of the current research.

Let $\mathcal{G}=\left[\left(A_{i}, \pi_{i}\right) ; i=1,2\right]$ be the two-party hybrid electoral competition game under uncertainty, where each party payoff function $\pi_{i}$ satisfies A1-A3.

Definition 1 A pure strategy Nash equilibrium of $\mathcal{G}$ is a strategy profile $\left(x_{1}^{*}, x_{2}^{*}\right) \in A$ such that, $\forall\left(x_{1}, x_{2}\right) \in A, \pi_{1}\left(x_{1}^{*}, x_{2}^{*}\right) \geq \pi_{1}\left(x_{1}, x_{2}^{*}\right)$ and $\pi_{2}\left(x_{1}^{*}, x_{2}^{*}\right) \geq \pi_{2}\left(x_{1}^{*}, x_{2}\right)$.

We denote $N E(\mathcal{G})$ the set of all PSNE of $\mathcal{G}$. For the particular case where $\chi_{1}=\chi_{2}=\infty$, that is, for the Downsian game, and for $\chi_{1}=\chi_{2}=0$, which represents the Wittman game, it is well known that this set is nonempty. We will not go further into the details of these results, except to mention that the interested reader can find a comprehensive analysis in Roemer [27]. 
On the other hand, in the hybrid case, where $\forall i=1,2, \chi_{i}>0$, the discontinuities of the payoff functions do not allow to apply the traditional analysis of equilibrium existence, based on the direct application of a fixed point theorem. However, it is possible to circumvent this difficulty by invoking recent sufficient conditions given by Reny [24]. To do that, we introduce the following notation and definitions. Let $d: \mathbb{R}^{k} \rightarrow \mathbb{R}_{+}$be the usual distance function on $\mathbb{R}^{k}$, and $B_{\delta}(y)=\left\{x \in \mathbb{R}^{k}: d(x, y)<\delta\right\}$ the open ball about $y$ with radius $\delta>0$.

Definition 2 Party 1 can secure a payoff $\alpha \in \mathbb{R}$ at $\left(x_{1}, x_{2}\right) \in A$ if there exists $\tilde{x}_{1} \in A_{1}$ and $\delta>0$ such that, $\forall x_{2}^{\prime} \in B_{\delta}\left(x_{2}\right), \pi_{1}\left(\tilde{x}_{1}, x_{2}^{\prime}\right) \geq \alpha$.

This definition can be extended in the obvious way to party 2 . In words, party $i$ can secure a payoff $\alpha$ at $x$ if it has a strategy that guarantees at least that payoff even if the other slightly deviates from $x$.

Definition $3 \mathcal{G}$ is payoff secure if $\forall x \in A$ and $\forall \epsilon>0$, each party $i$ can secure a payoff of $\pi_{i}(x)-\epsilon$ at $x$.

Payoff security requires that for every profile $x \in A$, each party has a strategy that virtually guarantees the payoff he receives at $x$ even if the other slightly deviates from $x$.

Let $\pi: A \rightarrow \mathbb{R}^{2}$ be the vector payoff function of $\mathcal{G}$, defined as, $\forall x \in A, \pi(x)=\left(\pi_{1}(x), \pi_{2}(x)\right)$. The graph of $\pi$ is a subset of $A \times \mathbb{R}^{2}$. That is, $g r(\pi)=\left\{(x, \pi) \in A \times \mathbb{R}^{2}: \forall i, \pi_{i}(x)=\pi_{i}\right\}$. Let $\operatorname{cl}(\operatorname{gr}(\pi))$ be the closure of the graph of the vector payoff function. ${ }^{14}$

Definition $4 \mathcal{G}$ is reciprocally upper semi-continuous if $\forall(\hat{x}, \hat{\pi}) \in \operatorname{cl}(\operatorname{gr}(\pi))$ such that $\forall i, \pi_{i}(\hat{x}) \leq$ $\hat{\pi}_{i}$, it follows that $\forall i, \pi_{i}(\hat{x})=\hat{\pi}_{i}$.

Reciprocally upper semi-continuity (r.u.s.c.) generalizes the condition introduced by Dasgupta and Maskin [7] that the sum of the players' payoffs be upper semi-continuous. In effect, suppose $\Pi$ is u.s.c. on $A$, and assume, by contradiction, there exists $(\bar{x}, \bar{\pi}) \in \operatorname{cl}(\operatorname{gr}(\pi))$ such that $\forall i, \pi_{i}(\bar{x}) \leq \bar{\pi}_{i}$, and $\pi(\bar{x}) \neq \bar{\pi}$. Then, $\pi_{1}(\bar{x})+\pi_{2}(\bar{x})<\bar{\pi}_{1}+\bar{\pi}_{2}$. Consider a sequence $\left(x_{1}^{n}, x_{2}^{n}\right) \subseteq A$ such that $\left(x_{1}^{n}, x_{2}^{n}\right) \rightarrow\left(\bar{x}_{1}, \bar{x}_{2}\right)$ and $\forall i$, $\lim \sup _{x^{n} \rightarrow \bar{x}} \pi_{i}\left(x_{1}^{n}, x_{2}^{n}\right)=\bar{\pi}_{i}$. Since $(\bar{x}, \bar{\pi}) \in \operatorname{cl}(\operatorname{gr}(\pi))$, this sequence always exists. Then, $\lim \sup _{x^{n} \rightarrow \bar{x}} \Pi\left(x^{n}\right)=\bar{\pi}_{1}+\bar{\pi}_{2}$. On the other hand, $\lim \sup _{x^{n} \rightarrow \bar{x}} \Pi\left(x^{n}\right) \leq$ $\pi_{1}(\bar{x})+\pi_{2}(\bar{x})$, because $\Pi$ is u.s.c. on $A$. Hence, $\bar{\pi}_{1}+\bar{\pi}_{2} \leq \pi_{1}(\bar{x})+\pi_{2}(\bar{x})$, a contradiction. Thus, if $\Pi$ is u.s.c., the game must be r.u.s.c. Now, we turn to the equilibrium analysis.

\section{$3 \quad$ Pure strategy analysis}

This section contains three main results. First of all, we prove in Section 3.1 that the hybrid election game is payoff secure and reciprocally upper semi-continuous. Then, in Section 3.2, we present an example which illustrates the fact that, with mixed motives, the unidimensional two-party electoral competition game does not always possess a Nash equilibrium in pure strategies. As we said in the Introduction, this problem has been already pointed out in Ball [4]. However, here we argue that, because Reny's conditions are satisfied, the nonexistence of a PSNE for all parametric conditions is a direct consequence of the violation of quasi-concavity. Finally, in Section 3.3 we show that non-convexities matter only if the relative interest in holding office is heterogeneous across parties (i.e. only if $\chi_{1} \neq \chi_{2}$ ). Otherwise, a pure strategy Nash equilibrium not only exists, but it is also unique (Section 3.4).

\footnotetext{
${ }^{14}$ Recall that $\left(x^{*}, \pi^{*}\right) \in \operatorname{cl}(\operatorname{gr}(\pi))$ if and only if $\forall \epsilon>0, B_{\epsilon}\left(x^{*}, \pi^{*}\right) \cap \operatorname{gr}(\pi) \neq \emptyset$.
} 


\subsection{Reny's conditions}

Let's start showing that $\mathcal{G}$ satisfies payoff security and r.u.s.c. These properties will be useful not only to understand why a PSNE does not always exist, but also to show in Section 4 that the mixed extension is better reply secure (yet to be defined).

Lemma $2 \mathcal{G}$ is payoff secure.

Proof. Suppose, by contradiction, $\exists \bar{x}=\left(\bar{x}_{1}, \bar{x}_{2}\right) \in A$ and $\bar{\epsilon}>0$ such that for some $i$ the payoff $\pi_{i}(\bar{x})-\bar{\epsilon}$ cannot be secured at $\bar{x}$. Without loss of generality, assume $i=1$. The fact that party 1 cannot secure $\pi_{1}(\bar{x})-\bar{\epsilon}$ at $\bar{x}$ implies that $\forall \tilde{x}_{1} \in A_{1}$, and $\forall \delta>0, \exists x_{2}^{\prime} \in B_{\delta}\left(\bar{x}_{2}\right)$ such that $\pi_{1}\left(\tilde{x}_{1}, x_{2}^{\prime}\right)<\pi_{1}\left(\bar{x}_{1}, \bar{x}_{2}\right)-\bar{\epsilon}$. If $\left(\bar{x}_{1}, \bar{x}_{2}\right) \notin D(A)$, then $\pi_{1}$ is continuous at $\bar{x}$. Therefore, $\exists \bar{\delta}>0$ such that $\forall x_{2}^{\prime} \in B_{\bar{\delta}}\left(\bar{x}_{2}\right), \pi_{1}\left(\bar{x}_{1}, x_{2}^{\prime}\right)>\pi_{1}\left(\bar{x}_{1}, \bar{x}_{2}\right)-\bar{\epsilon}$. That is, if $\bar{x}_{1} \neq \bar{x}_{2}$, we have a strategy for party $1, \bar{x}_{1}$, and an interval for $x_{2}$, determined by $\bar{\delta}>0$, which contradict our initial hypothesis. Hence, $\left(\bar{x}_{1}, \bar{x}_{2}\right) \in D(A)$, and $\pi_{1}(\bar{x})=\chi_{1} / 2$. If $\chi_{1}=0$, then $\pi_{1}$ is continuous on $A$, (see Roemer [26], Lemma 1), so that the previous argument goes through as before. Instead, if $\chi_{1}>0$, three cases are possible, depending on the position of $\bar{x}_{1}$ and $\theta^{1}$ over $[0,1]{ }^{15}$

Case 1: $\theta^{1}<\bar{x}_{1}<\theta_{m}$. Consider an alternative $\tilde{x}_{1} \in A_{1}, \tilde{x}_{1}>\bar{x}_{1}$, close enough to $\bar{x}_{1}$. Define $\bar{\delta}=d\left(\tilde{x}_{1}, \bar{x}_{1}\right)$ and $B_{\bar{\delta}}\left(\bar{x}_{2}\right)$. We choose $\tilde{x}_{1}$ so that $\theta^{1}<\bar{x}_{1}-\bar{\delta}$ and $\bar{x}_{1}+\bar{\delta}<\theta_{m}$. Since $\theta^{1}<\bar{x}_{1}<\theta_{m}$, this number always exists. By A1, $\forall x_{2}^{\prime} \in B_{\bar{\delta}}\left(\bar{x}_{2}\right), \pi_{1}\left(\tilde{x}_{1}, x_{2}^{\prime}\right)=p\left(\tilde{x}_{1}, x_{2}^{\prime}\right)\left(\chi_{1}-(1-\alpha) \bar{\delta}\right)$, where $\alpha \in(-1,1)$. Notice that $p$ is discontinuous at $\bar{x}$, so $p\left(\tilde{x}_{1}, x_{2}^{\prime}\right)$ is well above $1 / 2$. On the other hand, $p$ is bounded. Therefore, $\exists \bar{\delta}>0$ small enough such that $\forall x_{2}^{\prime} \in B_{\bar{\delta}}\left(\bar{x}_{2}\right)$ and $\forall \epsilon>0$, $\pi\left(\tilde{x}_{1}, x_{2}^{\prime}\right)>\chi_{1} / 2-\epsilon$, a contradiction. ${ }^{16}$

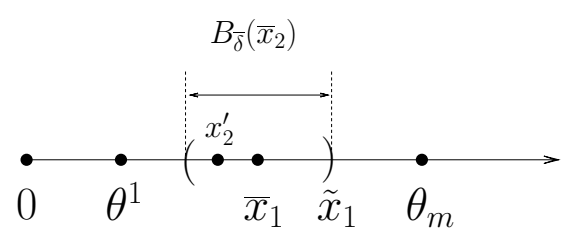

Case 1

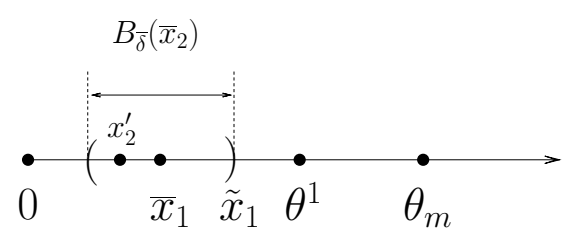

Case 2

Figure 2: Payoff security

Case 2: $\bar{x}_{1}<\theta^{1}<\theta_{m}$. Like in the previous case, consider $\tilde{x}_{1}>\bar{x}_{1}$ and $\bar{\delta}=d\left(\tilde{x}_{1}, \bar{x}_{1}\right)$, such that $\bar{x}_{1}+\bar{\delta}<\theta^{1}$. By A1, for all $x_{2}^{\prime} \in B_{\bar{\delta}}\left(\bar{x}_{2}\right), \pi_{1}\left(\tilde{x}_{1}, x_{2}^{\prime}\right)=p\left(\tilde{x}_{1}, x_{2}^{\prime}\right)\left((1-\alpha) \bar{\delta}+\chi_{1}\right), \alpha \in(-1,1)$. Again, $p\left(\tilde{x}_{1}, x_{2}^{\prime}\right)>1 / 2$. Moreover, $(1-\alpha) \bar{\delta}>0$. Hence, $\forall x_{2}^{\prime} \in B_{\bar{\delta}}\left(\bar{x}_{2}\right)$ and $\forall \epsilon>0, \pi\left(\tilde{x}_{1}, x_{2}^{\prime}\right)>\chi_{1} / 2-\epsilon$ : contradiction.

Case 3: $\theta^{1}<\bar{x}_{1}=\theta_{m}$. Since the probability of winning $p\left(x_{1}, x_{2}\right)$ is continuous at $\left(\theta_{m}, \theta_{m}\right)$, $\pi_{1}\left(\theta_{m}, \cdot\right)$ is continuous in $x_{2}$. Therefore, applying the argument based on continuity, we get the desired result.

\footnotetext{
${ }^{15} \mathrm{It}$ is easy to check that the remaining situations are variations of these three cases.

${ }^{16}$ Concretely, this is true for any $\bar{\delta}<\frac{\left(p\left(\tilde{x}_{1}, x_{2}^{\prime}\right)-1 / 2\right) \chi_{1}}{p\left(\tilde{x}_{1}, x_{2}^{\prime}\right)(1-\alpha)}$.
} 
Lemma 3 For all $\chi_{1}, \chi_{2} \in \mathbb{R}_{++}, \mathcal{G}$ is reciprocally upper semi-continuous.

Proof. Suppose, by contradiction, there exists $(\bar{x}, \bar{\pi}) \in \operatorname{cl}(\operatorname{gr}(\pi))$ such that $\forall i, \bar{\pi}_{i} \geq \pi_{i}(\bar{x})$, and $\left(\pi_{1}(\bar{x}), \pi_{2}(\bar{x})\right) \neq\left(\bar{\pi}_{1}, \bar{\pi}_{2}\right)$. Then, $\left(\bar{x}_{1}, \bar{x}_{2}\right) \in D(A)$. By hypothesis, there exists $i$ such that $\bar{\pi}_{i}>$ $\pi_{i}(\bar{x})$. Without loss of generality, suppose $i=1$. Consider a sequence $\left(x_{1}^{n}, x_{2}^{n}\right) \subseteq A$ such that $\left(x_{1}^{n}, x_{2}^{n}\right) \rightarrow\left(\bar{x}_{1}, \bar{x}_{2}\right)$ and $\forall i, \lim \sup _{x^{n} \rightarrow \bar{x}} \pi_{i}\left(x_{1}^{n}, x_{2}^{n}\right)=\bar{\pi}_{i}$. Since $(\bar{x}, \bar{\pi}) \in \operatorname{cl}(\operatorname{gr}(\pi))$, this sequence always exists. Notice that $\bar{\pi}_{1}=p\left(x_{1}^{n}, x_{2}^{n}\right) \chi_{1}$. On the other hand, $\pi_{1}\left(\bar{x}_{1}, \bar{x}_{2}\right)=1 / 2 \chi_{1}$. Therefore, $\left[1-p\left(x_{1}^{n}, x_{2}^{n}\right)\right] \chi_{2}<1 / 2 \chi_{2}$. But this implies that $\bar{\pi}_{2}<\pi_{2}(\bar{x})$ : contradiction. Hence, $\mathcal{G}$ is r.u.s.c.

By Lemma 1, $\mathcal{G}$ is also reciprocally upper semi-continuous if either $\chi_{1}=\chi_{2}=0$, or $\chi_{1}=\chi_{2}=\infty$, because $\Pi$ is continuous on $A$. On the contrary, it violates r.u.s.c. if, for example, one party is office motivated, and the other entirely ideological; i.e., if for some $i \neq j, \chi_{i}=0$ and $\chi_{j}=+\infty$. Intuitively, this happens because when a point on the diagonal is reached, parties' payoffs all jump in the same direction, except of course at the median ideal policy. ${ }^{17}$ Interestingly enough, our next proposition also indicates that, under the previous assumption, that is, if parties' relative interest in power is completely opposite, the hybrid election game not only violates r.u.s.c, but also a PSNE does not exist.

Assumption (A4) Party 1 is policy motivated (i.e., $\chi_{1}=0$ ), and represents a type $\theta^{1} \neq \theta_{m}$. Party 2 is office motivated (i.e., $\left.\chi_{2} \rightarrow+\infty\right)$.

Proposition 1 If $\mathcal{G}$ satisfies A1-A4, then $N E(\mathcal{G})=\emptyset$.

Proof. Fix the strategy profile $\left(\theta_{m}, \theta_{m}\right) \in A$, and consider party 1's deviation $x_{1} \neq \theta_{m}$. By A1, $F\left(S\left(x_{1}, \theta_{m}\right)\right)<1 / 2$. Then, $p\left(x_{1}, \theta_{m}\right)=1-H\left[1 / 2-F\left(S\left(x_{1}, \theta_{m}\right)\right)\right]<1 / 2=p\left(\theta_{m}, \theta_{m}\right)$. By continuity of $F$ and $H, \exists \delta>0$ such that $\forall x_{1} \in B_{\delta}\left(\theta_{m}\right), p\left(x_{1}, \theta_{m}\right)>0$. And again, by A1, for $\epsilon=d\left(\theta_{m}, \theta^{1}\right)$ and $\forall x_{1} \in B_{\epsilon}\left(\theta^{1}\right), \psi\left(x_{1}, \theta_{m} ; \theta^{1}\right)>0$. Hence, $\forall x_{1} \in B_{\epsilon}\left(\theta^{1}\right) \cap B_{\delta}\left(\theta_{m}\right), \pi_{1}\left(x_{1}, \theta_{m}\right)>0=\pi_{1}\left(\theta_{m}, \theta_{m}\right)$, implying that $\left(\theta_{m}, \theta_{m}\right) \notin N E(\mathcal{G})$.

Next, consider any $(x, x) \in D(A), x \neq \theta_{m}$. Let $\delta=d\left(x, \theta_{m}\right)$. By A1, $\forall \tilde{x} \in B_{\delta}\left(\theta_{m}\right), F(S(x, \tilde{x}))<$ $1 / 2$ and, therefore, $\pi_{2}(x, \tilde{x})=1-p(x, \tilde{x})>1 / 2=\pi_{2}(x, x)$. Thus, $(x, x) \notin N E(\mathcal{G})$. Finally, suppose there exists $\left(x_{1}, x_{2}\right) \in A-D(A)$ such that $\left(x_{1}, x_{2}\right) \in N E(\mathcal{G})$. Without loss of generality, let $\theta^{1}>\theta_{m}$. Then, $x_{1} \geq \theta_{m}$. Suppose not. If $x_{2}>x_{1}$, party 1 can deviate to $\tilde{x}_{1}=\theta_{m}$, and increase both $\psi\left(\cdot, x_{2}, \theta^{1}\right)$ and $p\left(\cdot, x_{2}\right)$. On the other hand, if $x_{2}<x_{1}$, the deviation $x_{2}^{\prime}=x_{1}$ increases party 2 's payoff. Therefore, $x_{1} \geq \theta_{m}$. Moreover, if $x_{1}=\theta_{m}$, then $\left(\theta_{m}, x_{2}\right) \notin N E(\mathcal{G})$, since 2 can deviate to $\theta_{m}$ and increase its probability of being in power. Thus, $x_{1}>\theta_{m}$. But then, for any $x_{2} \in[0,1]$, $x_{2} \neq x_{1}$, there exists $\delta>0$ such that $\forall \tilde{x}_{2} \in\left(x_{1}-\delta, x_{1}\right), p\left(x_{1}, \tilde{x}_{2}\right)<p\left(x_{1}, x_{2}\right)$, contradicting that $\left(x_{1}, x_{2}\right) \in N E(\mathcal{G}){ }^{18}$

Proposition 1 reveals a displeasing feature of unidimensional electoral competition. However, since the result is obtained under A4, which is an extreme hypothesis about parties' motives, one could initially think that the problem is created by that assumption. Unfortunately, this is not the case. To see this in more detail, in the next section we discuss the uniform distribution

\footnotetext{
${ }^{17}$ For instance, let $\chi_{1}=0$ and $\chi_{2} \rightarrow+\infty$, and consider $x^{n}=\left(x_{1}^{n}, x_{2}^{n}\right)=\left(\bar{x}, \bar{x}-\frac{1}{n}\right) \subseteq A$, where $n>0$ and $\theta_{m}<\bar{x}<\theta^{1}$. Then, party 1's payoff, $p\left(x_{1}^{n}, x_{2}^{n}\right) \psi\left(x_{1}^{n}, x_{2}^{n} ; \theta^{1}\right)$, is positive and converges to 0 as $n \rightarrow \infty$, while party 2 's payoff, $1-p\left(x_{1}^{n}, x_{2}^{n}\right)$, increases first above $1 / 2$, and then it drops down to $1 / 2$ when $(\bar{x}, \bar{x})$ is reached.

${ }^{18}$ That is, if $x_{1}>\theta_{m}$, party 2 would like to choose the largest platform that is just below $x_{1}$. However, since the policy space is a continuum, this value is not well defined.
} 
version of the hybrid election game. The example confirms that the message coming out from Proposition 1 is not an artifact of $\mathrm{A} 4$, but a more general feature of electoral competition. In particular, it points out that, if parties have heterogeneous interests in power, (i.e., if $\chi_{1} \neq \chi_{2}$ ), though not necessarily opposite, then the existence of a PSNE depends upon the relationship between the electoral uncertainty, the aggregate level of opportunism and its distribution among political parties.

\subsection{Uniform distribution}

Suppose $\xi$ is uniformly distributed on $[-\beta, \beta]$. Let $\theta^{1}<\theta_{m}<\theta^{2}$ and $\chi_{i}>0$ for all $i$. Consider an equilibrium candidate $\left(x_{1}^{*}, x_{2}^{*}\right)$ for this version of the hybrid game, with the property that $\theta^{1}<x_{1}^{*}<x_{2}^{*}<\theta^{2}$. Near the equilibrium, parties' payoffs can be rewritten as

$$
\begin{aligned}
& \pi_{1}\left(x_{1}, x_{2}\right)=p\left(x_{1}, x_{2}\right)\left(x_{2}-x_{1}+\chi_{1}\right), \\
& \pi_{2}\left(x_{1}, x_{2}\right)=\left(1-p\left(x_{1}, x_{2}\right)\right)\left(x_{2}-x_{1}+\chi_{2}\right),
\end{aligned}
$$

where $p\left(x_{1}, x_{2}\right)=\frac{1}{2 \beta}\left[F\left(S\left(x_{1}, x_{2}\right)\right)-\frac{1}{2}+\beta\right]$ if $x_{1} \neq x_{2}$, and $p\left(x_{1}, x_{2}\right)=1 / 2$ otherwise. Taking the first order conditions, we have

$$
\begin{aligned}
f(\hat{x})\left(x_{2}-x_{1}+\chi_{1}\right)-2 \beta & =2 F\left(\frac{x_{1}+x_{2}}{2}\right)-1, \\
-f(\hat{x})\left(x_{2}-x_{1}+\chi_{2}\right)+2 \beta & =2 F\left(\frac{x_{1}+x_{2}}{2}\right)-1 .
\end{aligned}
$$

Assuming that $\theta$ is also uniformly distributed on $[0,1]$ and solving (2), it follows that $x_{1}^{*}=$ $1 / 2-\beta+\chi_{1} / 2$ and $x_{2}^{*}=1 / 2+\beta-\chi_{2} / 2$. It is easy to verify that, if $2 \beta-1<\chi_{i}<2 \beta$, then the pair of platforms

$$
\left(x_{1}^{*}, x_{2}^{*}\right)=\left(\frac{1}{2}-\beta+\frac{\chi_{1}}{2}, \frac{1}{2}+\beta-\frac{\chi_{2}}{2}\right)
$$

constitutes indeed a Nash equilibrium of the game. The first condition, $2 \beta-1<\chi_{i}$, ensures that $x_{1}^{*}>0$ and $x_{2}^{*}<1$. On the other hand, in order to guarantee that $x_{1}^{*}<x_{2}^{*}$, we need

$$
\chi_{1}+\chi_{2}<4 \beta
$$

which is fulfilled if $\chi_{i}<2 \beta$ for all $i$. In fact, this implies that $x_{1}^{*}<\theta_{m}<x_{2}^{*}{ }^{19}$

Contrary, if (3) is not satisfied, that is, if the aggregate level of opportunism, measured by $\chi_{1}+\chi_{2}$, is relatively high compared with the electoral uncertainty, represented by $\beta$, then $\left(x_{1}^{*}, x_{2}^{*}\right)$ is not an equilibrium. Is there any other candidate? Since $\theta^{1}<\theta_{m}<\theta^{2}$, it is clear that $x_{1}>x_{2}$ cannot be a Nash equilibrium either. Hence, the only remaining possibility is $x_{1}=x_{2}$.

Assume that $\chi_{1}+\chi_{2} \geq 4 \beta$. Consider first the case where parties have homogeneous motivations, i.e. $\chi_{1}=\chi_{2}=\chi$. Take the pair $\left(x_{1}, x_{2}\right)=\left(\theta_{m}, \theta_{m}\right)$ as the equilibrium candidate. Then $\forall i, \pi_{i}\left(\theta_{m}, \theta_{m}\right)=\chi / 2$. Consider party 1 's deviation to $x_{1}^{\prime}=\theta_{m}-\delta, \delta>0$. (Recall that deviations to the right of $\theta_{m}$ or below $\theta^{1}$ are not profitable for 1$)$. Then,

$$
\pi_{1}\left(x_{1}^{\prime}, \theta_{m}\right)=\frac{-\delta^{2}}{4 \beta}+\left(\frac{1}{2}-\frac{\chi_{1}}{4 \beta}\right) \delta+\frac{\chi_{1}}{2} .
$$

\footnotetext{
${ }^{19}$ Second order conditions also hold, because $\forall i, \partial^{2} \pi_{i} / \partial x_{i}^{2}=-2<0$.
} 
This deviation is profitable, that is, $\pi_{1}\left(x_{1}^{\prime}, \theta_{m}\right)>\pi_{1}\left(\theta_{m}, \theta_{m}\right)$, if and only if $\delta<2 \beta-\chi$. But, since $\chi \geq 2 \beta$, this requires $\delta<0$, a contradiction.

In the same way, consider party 2's deviation to $x_{2}^{\prime}=\theta_{m}+\delta, \delta>0$. Following the same reasoning, $\pi_{2}\left(\theta_{m}, x_{2}^{\prime}\right)>\pi_{2}\left(\theta_{m}, \theta_{m}\right)$ if and only if $\delta<2 \beta-\chi$, which contradicts again the initial hypothesis. Therefore, since 2 cannot improve its payoff by deviating to the left of $\theta_{m}$ or above $\theta^{2}$, it follows that $\left(\theta_{m}, \theta_{m}\right)$ is indeed a pure strategy Nash equilibrium of $\mathcal{G} .^{20}$

What about the other case in which $\chi_{1} \neq \chi_{2}$ ? Is $\left(\theta_{m}, \theta_{m}\right)$ a PSNE? As before, a deviation is profitable for party $i$ only if $\delta<2 \beta-\chi_{i}$. Suppose $\chi_{1}=3 \beta / 2$ and $\chi_{2}=5 \beta / 2$. Note that we still have $\chi_{1}+\chi_{2} \geq 4 \beta$. Therefore, there is no PSNE with $x_{1}<x_{2}$. Moreover, for $\beta>1 / 2$ and $\delta<1 / 4$, any deviation $x_{1}^{\prime}=\theta_{m}-\delta$ is such that $\pi_{1}\left(x_{1}^{\prime}, \theta_{m}\right)>\pi_{1}\left(\theta_{m}, \theta_{m}\right)$. So, $\left(\theta_{m}, \theta_{m}\right)$ is not a Nash equilibrium either. Finally, since $x_{1}>x_{2}$ and $x_{1}=x_{2} \neq \theta_{m}$ cannot be equilibrium strategies, it follows that, for these values of $\chi_{1}, \chi_{2}$ and $\beta$, the hybrid election game does not possess a PSNE.

\subsection{Equilibrium existence}

The previous section offers interesting insights about electoral competition. First, it shows that, although $\chi_{i}>0$ creates discontinuities in parties' payoffs, this does not necessarily preclude the existence of a PSNE. At least in the uniform distribution case, no matter how different are $\chi_{1}$ and $\chi_{2}$, if the aggregate level of opportunism is low relative to the electoral uncertainty, then a PSNE always exists. In the example, sufficient conditions are $2 \beta-1<\chi_{i}<2 \beta$ for all $i=1,2$. Moreover, in that case the equilibrium is such that $x_{1}^{*}<x_{2}^{*}$. That is, equilibrium platforms do not converge, though $x_{i}^{*} \rightarrow \theta_{m}$ as $\chi_{i} \rightarrow 2 \beta$.

On the other hand, when the aggregate level of opportunism is high, (that is, when (3) does not hold), and it is relatively more concentrated in one party, then a PSNE may not exist. In the example, this occurs if (i) $\chi_{1}+\chi_{2} \geq 4 \beta$, and (ii) $\chi_{1}<2 \beta$. This result is driven by two forces. On one hand, the relatively more opportunistic party precludes equilibria with policy differentiation. On the other, the relatively more ideological party blocks, due to its excessive policy orientation, a Nash equilibrium at the median position.

Remarkably, in our model the nonexistence of a PSNE is not related with the dimensionality of the policy space, nor the heterogeneity of interests in the electorate, which are usually viewed in the literature as the main causes behind existence problems. ${ }^{21}$ In this regard, notice that the model satisfies two extremely nice properties, frequently invoked to guarantee equilibria: (i) the policy space is unidimensional; and (ii) voters' preferences are single-peaked and symmetric, so that they admit the existence of a unique Condorcet winner.

Moreover, contrary to Ball [4], we argue that it is not directly related with neither, the discontinuities of the probability of winning function, nor the mixed motivations. As Lemmas 2 and 3 already showed, $\mathcal{G}$ is payoff secure and r.u.s.c. for all $\chi_{1}, \chi_{2} \in \mathbb{R}_{++}$. Thus, following Reny's [24] Corollary 3.3, our game should always possess a PSNE, provided that each party's payoff function is quasi-concave in its own strategy. This shows that the real blame for the failure of the hybrid electoral competition game to possess a Nash equilibrium in pure strategies for all parametric

\footnotetext{
${ }^{20}$ That is, provided that parties' benefits of holding office are sufficiently high relative to the uncertainty, the convergence to the median ideal policy still holds under mixed motivations. This is consistent with Calvert [6], who showed that "small departures" from the classic assumptions (i.e., office motivation and certainty) lead to only "small departures" from convergence.

${ }^{21}$ As we immediately show, it is due to the heterogeneity of interests among parties!
} 
conditions can be fully assigned to the violation of quasi-concavity. ${ }^{22}$ Example 2 below further illustrates this point.

Example 2 Consider the uniform case studied before. Fix $\beta=1 / 2$ and $\bar{x}_{2}=\frac{49}{100}$. Assume $\chi_{1}=\frac{3}{4}$ and $\theta^{1}=\frac{1}{4}$. Under these conditions, party 1's conditional payoff can be written as

$$
\pi_{1}\left(x_{1}, \bar{x}_{2}\right)= \begin{cases}\frac{1}{2}\left(x_{1}+\frac{49}{100}\right)\left(x_{1}+\frac{37}{50}\right) & \text { if } 0 \leq x_{1}<\frac{1}{4} \\ \frac{1}{2}\left(x_{1}+\frac{49}{100}\right)\left(\frac{31}{25}-x_{1}\right) & \text { if } \frac{1}{4} \leq x_{1}<\frac{49}{100} \\ \frac{3}{8} & \text { if } x_{1}=\frac{49}{100} \\ \frac{1}{2}\left(\frac{151}{100}-x_{1}\right)\left(\frac{31}{25}-x_{1}\right) & \text { if } \frac{49}{100}<x_{1} \leq 1 .\end{cases}
$$

Notice that $\pi_{1}(3 / 8,49 / 100)=0.3741125, \quad \lim _{x_{1} \rightarrow-49 / 100} \pi_{1}\left(x_{1}, \bar{x}_{2}\right)=0.3675$ and $\lim _{x_{1} \rightarrow+49 / 100} \pi_{1}\left(x_{1}, \bar{x}_{2}\right)=0.3825$. Therefore, $\pi_{1}\left(\cdot, \bar{x}_{2}\right)$ is not quasi-concave in $x_{1}$, since we can take for instance the convex combination $x_{1}^{\lambda}=(1-\lambda) \frac{3}{8}+\lambda \frac{491}{1000}$ and get $\pi_{1}\left(x_{1}^{\lambda}, 49 / 100\right)<$ $\min \left\{\pi_{1}(3 / 8,49 / 100), \pi_{1}(491 / 1000,49 / 100)\right\}$ for all $\lambda \in(0,1)$.

Regarding equilibrium existence, however, note that the non-convexities of the conditional payoffs are important only if the relative interest in power is heterogeneous across parties. In effect, if parties have heterogeneous motivations, then there is no reason to believe they will locate at the median, or on their own ideological side. Contrary, in order to undercut the rival's proposal, they may find profitable to move their platforms to the other party's ideological arena. So, in principle, the game could reach a point on the diagonal where the discontinuities and non-convexities play an active role against existence. In fact, this is not just a possibility. As we saw, it happens indeed under certain parameters' values.

By contrast, if motivations are homogeneous, (i.e., if $\chi_{1}=\chi_{2}$ ), parties never find it profitable to behave in the way depicted above. Therefore, under the conditions specified below, the unidimensional, two-party electoral competition game always possesses a Nash equilibrium in pure strategies. For the uniform distribution, this was already illustrated in Section 3.2, since $\chi_{1}=\chi_{2}$ led to an equilibrium where both parties converge to the median ideal policy, or to an equilibrium where each candidate plays a pure strategy in its own ideological side. Now, we generalize the result for any probability distribution.

To do that, we introduce the following two additional assumptions.

Assumption (A5) $\theta^{1}<\theta_{m}<\theta^{2}$.

In words, A5 simply says that party 1 is left-oriented and party 2 right-oriented. As Calvert [6] reckoned, this case where parties' policy goals are opposed to one another relative to the electorate seems the important case to focus on.

Assumption (A6) $\log \left(p\left(x_{1}, x_{2}\right)\right)$ and $\log \left(1-p\left(x_{1}, x_{2}\right)\right)$ are concave in $x_{1}$ and $x_{2}$, respectively.

The last assumption is certainly not nice. However, it is standard in the literature on electoral competition with ideological parties. The point is that, as it happens in the probabilistic version of the Wittman game, no simple conditions are known to ensure the concavity of the probability of winning function. For more on this, see the discussion in Roemer [27].

Now we state and prove our first theorem.

\footnotetext{
${ }^{22}$ Wittman [30] showed that conditional payoffs are concave. However, the argument rested on the assumption that the probability of winning the election is continuous, which is obviously not true.
} 
Theorem 1 If $\mathcal{G}$ verifies $A 1-A 3, A 5$ and $A 6$ and $\chi_{1}=\chi_{2}$, then $N E(\mathcal{G}) \neq \emptyset$.

Proof. Consider the following restricted game $\hat{\mathcal{G}}=\left[\left(\hat{A}_{i}, \log \left(\left.\pi_{i}\right|_{\hat{A}}\right)\right) ; i=1,2\right]$, where $\hat{A}_{1}=\left[0, \theta_{m}\right]$, $\hat{A}_{2}=\left[\theta_{m}, 1\right]$, and $\left.\pi_{i}\right|_{\hat{A}}$ represents the restriction of $\pi_{i}$ over $\hat{A}=\hat{A}_{1} \times \hat{A}_{2}$. Note that, $\hat{A}_{i}$ is nonempty, compact and convex, and $\log \left(\left.\pi_{i}\right|_{\hat{A}}\right)$ is continuous, because $p\left(x_{1}, x_{2}\right)$ is continuous on $\hat{A}$. Moreover,

$$
\begin{aligned}
& \log \left(\left.\pi_{1}\right|_{\hat{A}}\left(x_{1}, x_{2}\right)\right)=\log \left(p\left(x_{1}, x_{2}\right)\right)+\log \left(\psi\left(x_{1}, x_{2} ; \theta^{1}\right)+\chi_{1}\right), \\
& \log \left(\left.\pi_{2}\right|_{\hat{A}}\left(x_{1}, x_{2}\right)\right)=\log \left(1-p\left(x_{1}, x_{2}\right)\right)+\log \left(\psi\left(x_{2}, x_{1} ; \theta^{2}\right)+\chi_{2}\right) .
\end{aligned}
$$

Hence, for each fixed value of $x_{j}, \log \left(\left.\pi_{i}\right|_{\hat{A}}\right), i \neq j$, is concave on $x_{i}$. And using Kakutani's fixed point theorem, it follows that there exists a strategy profile $\left(x_{1}^{*}, x_{2}^{*}\right) \in \hat{A}$ such that $\forall\left(x_{1}, x_{2}\right) \in \hat{A}$, $\left.\pi_{1}\right|_{\hat{A}}\left(x_{1}^{*}, x_{2}^{*}\right) \geq\left.\pi_{1}\right|_{\hat{A}}\left(x_{1}, x_{2}^{*}\right)$ and $\left.\pi_{2}\right|_{\hat{A}}\left(x_{1}^{*}, x_{2}^{*}\right) \geq\left.\pi_{2}\right|_{\hat{A}}\left(x_{1}^{*}, x_{2}\right)$. Thus, $\left(x_{1}^{*}, x_{2}^{*}\right)$ is a PSNE of $\hat{\mathcal{G}}$.

Next, we show that $\left(x_{1}^{*}, x_{2}^{*}\right)$ is also a PSNE of the original game $\mathcal{G}=\left[\left(A_{i}, \pi_{i}\right) ; i=1,2\right]$. To do that, we expand the set of strategies of both parties, and we study the incentives for unilateral deviations from the equilibrium candidate. For brevity, with carry out the analysis for party 1, but a similar argument also applies to the other party. There are two cases to consider, depending on the features of the equilibrium in $\hat{\mathcal{G}}$.

Case 1. If $x_{1}^{*}=x_{2}^{*}=\theta_{m}$, then it is immediate to see that there is no deviation $\tilde{x}_{1}>\theta_{m}$ such that $\pi_{1}\left(\tilde{x}_{1}, \theta_{m}\right)>\pi_{1}\left(\theta_{m}, \theta_{m}\right)=\chi_{1} / 2$. In effect, this would require $\left(p\left(\tilde{x}_{1}, \theta_{m}\right)-1 / 2\right) \chi_{1}+p\left(\tilde{x}_{1}, \theta_{m}\right)\left(\theta_{m}-\right.$ $\left.\tilde{x}_{1}\right)>0$. However, $p\left(\tilde{x}_{1}, \theta_{m}\right)<1 / 2, \theta_{m}<\tilde{x}_{1}$, and $\chi_{1} \geq 0$. Therefore, such alternative $\tilde{x}_{1}$ does not exist, and $\left(\theta_{m}, \theta_{m}\right) \in N E(\mathcal{G})$.

Case 2. If $x_{1}^{*}<x_{2}^{*}$, then three cases are possible. Suppose first $x_{1}^{*}<\theta_{m}=x_{2}^{*}$. Then, party 1's conditional payoff $\log \left(\pi_{1}\left(x_{1}, \theta_{m}\right)\right)$ is continuous and concave on $A_{1}$, and

$$
\arg \max _{A_{1}} \pi_{1}\left(x_{1}, x_{2}^{*}\right)=x_{1}^{*}
$$

That is, party 1's best response to $\theta_{m}$ does not change in going from $\hat{A}_{1}=\left[0, \theta_{m}\right]$ to $A_{1}=[0,1]$. Similarly, if $x_{1}^{*}<\theta_{m}<x_{2}^{*}$, then $(*)$ holds, because $\log \left(\pi_{1}\left(x_{1}, x_{2}^{*}\right)\right)$ is continuous everywhere except at $x_{2}^{*}$, and it drops down to the right of $x_{2}^{*}$. Party 1's conditional payoff is not longer concave, but it is strictly quasi-concave, so that its unique maximum on $A_{1}$ coincides again with its restricted maximum on $\hat{A}_{1}$.

Finally, assume $x_{1}^{*}=\theta_{m}<x_{2}^{*}$. In this case, we cannot conclude that $(*)$ holds, because $x_{1}^{*}$ is a corner solution in the restricted game. Therefore, $\pi_{1}\left(x_{1}, x_{2}^{*}\right)$ could increase to the right of $\theta_{m}$. However, we claim that such a pair of strategies cannot be a PSNE of $\hat{\mathcal{G}}$. Instead, suppose $\left(x_{1}^{*}, x_{2}^{*}\right) \in N E(\hat{\mathcal{G}})$, where $N E(\hat{\mathcal{G}})$ is the set of PSNE of $\hat{\mathcal{G}}$. Differentiating $\pi_{i}$ with respect to $x_{i}$ and evaluating it at $\left(x_{1}^{*}, x_{2}^{*}\right)$, we have that

$$
\frac{1}{2}\left(x_{2}^{*}-x_{1}^{*}+\chi\right) h\left(\frac{1}{2}-F\left(\frac{x_{1}^{*}+x_{2}^{*}}{2}\right)\right) f\left(\frac{x_{1}^{*}+x_{2}^{*}}{2}\right) \geq p\left(x_{1}^{*}, x_{2}^{*}\right)
$$

and

$$
\frac{1}{2}\left(x_{2}^{*}-x_{1}^{*}+\chi\right) h\left(\frac{1}{2}-F\left(\frac{x_{1}^{*}+x_{2}^{*}}{2}\right)\right) f\left(\frac{x_{1}^{*}+x_{2}^{*}}{2}\right)=1-p\left(x_{1}^{*}, x_{2}^{*}\right) .
$$

But $p\left(x_{1}^{*}, x_{2}^{*}\right)>1 / 2$. Hence, these conditions cannot be simultaneously satisfied. This complete the proof. 
The result above generalizes the well known existence results corresponding to the Downsian and the Wittman electoral game. It clearly shows that the failure of the hybrid election game to always possess a PSNE does not lie behind the discontinuities of the payoff functions nor the mixed motivation assumption, but on parties' heterogeneous interests in power. Instead, in the homogeneous case, if parties locate on the diagonal, it must be at the median ideal policy. Hence, the discontinuities and non-convexities of the payoffs do not play a major role. This explain why "positive results" predominate all around Figure 1, where parties' (homogeneous) motivations are represented in the width of the rectangle.

As Section 3.2 suggests, however, things become much more complicated when we add a third dimension into the analysis, differentiating party 1's motivation from those of party 2 . To deal with these cases, in Section 4 we analyze whether the mixed extension of the hybrid election game possesses a Nash equilibrium. Before that, however, we close this section examining the number of PSNE in the homogeneous case.

\subsection{Uniqueness}

Having shown that the homogeneous version of the hybrid electoral competition game always has a PSNE, we can now ask about the cardinality of $N E(\mathcal{G})$. To do that, we assume until the end of this section that the hypotheses of Theorem 1 hold.

Proposition 2 Suppose the hybrid election game $\mathcal{G}$ satisfies A1-A3, A5 and A6, and let $\chi_{1}=\chi_{2}=$ $\chi$. Then, $N E(\mathcal{G})$ is a singleton.

The proof of Proposition 2 rests on three results. The first one says that, if the hybrid game has a PSNE on the diagonal, it must be at the median ideal policy, so that parties' payoffs around the equilibrium are always continuous and differentiable.

Lemma 4 If there exists $\left(x_{1}^{*}, x_{2}^{*}\right) \in N E(\mathcal{G})$ such that $x_{1}^{*}=x_{2}^{*}$, then $\left(x_{1}^{*}, x_{2}^{*}\right)=\left(\theta_{m}, \theta_{m}\right)$.

Proof. Without loss of generality, suppose there exists $\left(x_{1}^{*}, x_{2}^{*}\right) \in N E(\mathcal{G})$ such that $x_{1}^{*}=x_{2}^{*}<\theta_{m}$. Then, $\pi_{2}\left(x_{1}^{*}, x_{2}^{*}\right)=1 / 2 \chi$. Consider $x_{2}^{\prime}=x_{2}^{*}+\epsilon$, where $\epsilon>0$ and $x_{2}^{*}<x_{2}^{\prime}<\theta_{m}$. It is easy to see that $\pi_{2}\left(x_{1}^{*}, x_{2}^{\prime}\right)=\left(1-p\left(x_{1}^{*}, x_{2}^{\prime}\right)\right)(\chi+\epsilon)$. Moreover, since $1-p\left(x_{1}^{*}, x_{2}^{\prime}\right)>1 / 2$ and $\epsilon>0$, $\pi_{2}\left(x_{1}^{*}, x_{2}^{\prime}\right)>\pi_{2}\left(x_{1}^{*}, x_{2}^{*}\right)$, contradicting that $\left(x_{1}^{*}, x_{2}^{*}\right) \in N E(\mathcal{G})$.

Consider again the restricted game $\hat{\mathcal{G}}=\left[\left(\hat{A}_{i}, \log \left(\left.\pi_{i}\right|_{\hat{A}}\right)\right) ; i=1,2\right]$ introduced in the proof of Theorem 1, and denote $N E(\hat{\mathcal{G}})$ the set of PSNE of $\hat{\mathcal{G}}$. Our second result says that every PSNE of the hybrid game is also a Nash equilibrium of $\hat{\mathcal{G}}$.

Lemma $5 N E(\mathcal{G}) \subseteq N E(\hat{\mathcal{G}})$.

Proof. Assume, by contradiction, $\exists\left(x_{1}^{*}, x_{2}^{*}\right) \in N E(\mathcal{G})$ such that $\left(x_{1}^{*}, x_{2}^{*}\right) \notin N E(\hat{\mathcal{G}})$. Then, either $x_{1}^{*} \in\left(\theta_{m}, 1\right]$, or $x_{2}^{*} \in\left[0, \theta_{m}\right)$ or both. Without loss of generality, suppose $x_{1}^{*}>\theta_{m}$. By Lemma 4 , two cases are possible.

Case 1: $x_{2}^{*}<x_{1}^{*}$. Then, $x_{2}^{*} \geq \theta_{m}$, and $\pi_{1}\left(x_{1}^{*}, x_{2}^{*}\right)=p\left(x_{1}^{*}, x_{2}^{*}\right)\left(x_{2}^{*}-x_{1}^{*}+\chi\right)$. Moreover, since $p\left(x_{1}^{*}, x_{2}^{*}\right)<1 / 2$ and $x_{2}^{*}<x_{1}^{*}$, it follows that $\left[p\left(x_{1}^{*}, x_{2}^{*}\right)-1 / 2\right] \chi+p\left(x_{1}^{*}, x_{2}^{*}\right)\left[x_{2}^{*}-x_{1}^{*}\right]<0$. That is, $\pi_{1}\left(x_{1}^{*}, x_{2}^{*}\right)<1 / 2 \chi$. Therefore, party 1 can profitably deviate to $x_{1}^{\prime}=x_{2}^{*}$, a contradiction. 
Case 2: $x_{2}^{*}>x_{1}^{*}$. First, note that $x_{2}^{*} \leq \theta^{2}$. Therefore, around the equilibrium, $\pi_{1}\left(x_{1}, x_{2}\right)=$ $p\left(x_{1}, x_{2}\right)\left(x_{2}-x_{1}+\chi\right)$ and $\pi_{2}\left(x_{1}, x_{2}\right)=\left(1-p\left(x_{1}, x_{2}\right)\right)\left(x_{2}-x_{1}+\chi\right)$. Differentiating $\pi_{i}\left(x_{1}, x_{2}\right)$ with respect to $x_{i}$, and taking its value at $\left(x_{1}^{*}, x_{2}^{*}\right)$, we have that

$$
\begin{array}{r}
\frac{1}{2}\left(x_{2}^{*}-x_{1}^{*}+\chi\right) h\left(\frac{1}{2}-F\left(\frac{x_{1}^{*}+x_{2}^{*}}{2}\right)\right) f\left(\frac{x_{1}^{*}+x_{2}^{*}}{2}\right)-p\left(x_{1}^{*}, x_{2}^{*}\right)=0 \\
-\frac{1}{2}\left(x_{2}^{*}-x_{1}^{*}+\chi\right) h\left(\frac{1}{2}-F\left(\frac{x_{1}^{*}+x_{2}^{*}}{2}\right)\right) f\left(\frac{x_{1}^{*}+x_{2}^{*}}{2}\right)-\left(1-p\left(x_{1}^{*}, x_{2}^{*}\right)\right)=0,
\end{array}
$$

where the right hand side of (4), (respectively, (5)) follows from the first order conditions, because $x_{i}^{*}$ is an interior maximum of $\pi_{i}\left(\cdot, x_{j}^{*}\right)$. However, assuming that (4) holds, note that (5) cannot be true. If $p\left(x_{1}^{*}, x_{2}^{*}\right)<1$, then the left hand side of (5) is negative. On the other hand, if $p\left(x_{1}^{*}, x_{2}^{*}\right)=1$, then $H\left(\frac{1}{2}-F\left(\frac{x_{1}^{*}+x_{2}^{*}}{2}\right)\right)=0 \Rightarrow h\left(\frac{1}{2}-F\left(\frac{x_{1}^{*}+x_{2}^{*}}{2}\right)\right)=0$, because $\frac{1}{2}-F\left(\frac{x_{1}^{*}+x_{2}^{*}}{2}\right)<-\beta$. So, substituting this into (4), we have that $-1=0$, a contradiction.

Finally, let's show that $\hat{\mathcal{G}}$ has a unique PSNE. As we will immediately see, the proof exploits that this game is continuous and concave, with unidimensional strategy sets, which makes relatively simple to prove that the best reply correspondence is a contraction mapping.

Lemma $6 \# N E(\hat{\mathcal{G}})=1$.

Proof. For all $i \neq j$, and $x_{j} \in \hat{A}_{j}$, let $r_{i}\left(x_{j}\right)=\left\{\tilde{x}_{i} \in \hat{A}_{i}: \tilde{x}_{i} \in \arg \max _{x_{i} \in \hat{A}_{i}} \log \left(\left.\pi_{i}\right|_{\hat{A}}\left(x_{i}, x_{j}\right)\right)\right\}$ denote party $i$ 's best replies to platform $x_{j}$. The properties of $\hat{\mathcal{G}}$ ensures that $r_{i}\left(x_{j}\right)$ is a singleton. Abusing a bit the notation, let us refer to $r_{i}(\cdot)$ as the best reply function of $i$, and to $r(\cdot)=$ $\left(r_{1}(\cdot), r_{2}(\cdot)\right)$ as the best reply mapping of $\hat{\mathcal{G}}$, understanding that, for each $\left(x_{1}, x_{2}\right) \in \hat{A}, r\left(x_{1}, x_{2}\right)=$ $r_{1}\left(x_{2}\right) \times r_{2}\left(x_{1}\right)$.

We want to prove that $r(\cdot)$ is a contraction mapping. That is, we want to show that $r(\cdot)$ has a unique fixed point, which is also the unique PSNE of $\hat{\mathcal{G}}$. Since $\hat{A}_{i}$ is a closed and convex subset of real line, a sufficient condition for $r(\cdot)$ to be a contraction is, (Vives [29], pp. 47), $\forall i \neq j$, and $\forall\left(x_{i}, x_{j}\right) \in \hat{A}$,

$$
\frac{\partial^{2} \log \left(\left.\pi_{i}\right|_{\hat{A}}\left(x_{i}, x_{j}\right)\right)}{\partial x_{i}^{2}}+\left|\frac{\partial^{2} \log \left(\left.\pi_{i}\right|_{\hat{A}}\left(x_{i}, x_{j}\right)\right)}{\partial x_{i} \partial x_{j}}\right|<0 .
$$

Moreover, since for every pair $\left(x_{1}, x_{2}\right) \in \hat{A}$, we have that $r_{1}\left(x_{2}\right) \geq \theta^{1}$ and $r_{2}\left(x_{1}\right) \leq \theta^{2}$, we can restrict our attention to the subset of strategy profiles in $\left[\theta^{1}, \theta_{m}\right] \times\left[\theta_{m}, \theta^{2}\right]$.

Let us now check that $\log \left(\left.\pi_{1}\right|_{\hat{A}}\right)$ satisfies $(6) .{ }^{23}$ Note first that

$$
\frac{\partial^{2} \log \left(\left.\pi_{1}\right|_{\hat{A}}\left(x_{1}, x_{2}\right)\right)}{\partial x_{1}^{2}}=\frac{\partial^{2} \log \left(p\left(x_{1}, x_{2}\right)\right)}{\partial x_{1}^{2}}-\frac{1}{\left[\psi\left(x_{1}, x_{2} ; \theta^{1}\right)+\chi\right]^{2}},
$$

and

$$
\frac{\partial^{2} \log \left(\left.\pi_{1}\right|_{\hat{A}}\left(x_{1}, x_{2}\right)\right)}{\partial x_{1} \partial x_{2}}=\frac{\partial^{2} \log \left(p\left(x_{1}, x_{2}\right)\right)}{\partial x_{1} \partial x_{2}}+\frac{1}{\left[\psi\left(x_{1}, x_{2} ; \theta^{1}\right)+\chi\right]^{2}} .
$$

Moreover, it is easy to prove that $\frac{\partial^{2} \log \left(p\left(x_{1}, x_{2}\right)\right)}{\partial x_{1}^{2}}=\frac{\partial^{2} \log \left(p\left(x_{1}, x_{2}\right)\right)}{\partial x_{1} \partial x_{2}}$. Hence, if (8) is negative, (6) becomes $-2 /\left[\psi\left(x_{1}, x_{2} ; \theta^{1}\right)+\chi\right]^{2}$, which is obviously smaller than zero. Alternatively, if (8)

\footnotetext{
${ }^{23}$ The $\operatorname{argument}$ for $\log \left(\left.\pi_{2}\right|_{\hat{A}}\right)$ is similar.
} 
is nonnegative, then (6) is simply $2 \frac{\partial^{2} \log \left(p\left(x_{1}, x_{2}\right)\right)}{\partial x_{1}^{2}}$, which is also negative by A6. Repeating the reasoning for party 2's payoff, it follows that $r(\cdot)$ is a contraction. Therefore, it has a unique fixed point.

Proof of Proposition 2. Immediate from Lemmas 5 and 6.

\section{Mixed strategy analysis}

Assume now parties are allowed to randomize over campaign platforms. For each party $i=1,2$, let $\Delta\left(A_{i}\right)$ be the space of all (Borel) probability measures on $A_{i}=[0,1]$, and denote $\Delta(A)=$ $\Delta\left(A_{1}\right) \times \Delta\left(A_{2}\right)$. A mixed strategy for $i$ is a probability measure $\mu_{i} \in \Delta\left(A_{i}\right)$. As usual, we say that $\mu_{i}$ has support $\operatorname{supp}\left(\mu_{i}\right) \subseteq A_{i}$ if party $i$ assigns probability one over the set $\operatorname{supp}\left(\mu_{i}\right)$ under $\mu_{i}$, and we indicate by $\mathcal{A}\left(\mu_{i}\right)=\left\{x \in[0,1]: \mu_{i}(\{x\})>0\right\}$ the set of atoms of $\mu_{i}$. In what follows, we endow $\Delta\left(A_{i}\right)$ with the topology of weak convergence. That is, we say that a sequence of measures $\left\{\mu_{i}^{n}\right\} \subseteq \Delta\left(A_{i}\right)$ converges to $\mu_{i} \in \Delta\left(A_{i}\right)$ if for every real valued and continuous function $g$ defined on $A_{i}, \int_{A_{i}} g\left(x_{i}\right) d \mu_{i}^{n} \rightarrow \int_{A_{i}} g\left(x_{i}\right) d \mu_{i}$.

Since the discontinuities of parties' payoffs is the major obstacle to prove the existence of a PSNE in $\mathcal{G}$, before continuing it is necessary to show that each $\pi_{i}$ is a Borel measurable function on $A$, so that it makes sense to talk about mixed strategies and the expected value of $\pi_{i}$ under $\mu=\left(\mu_{1}, \mu_{2}\right) \in \Delta(A)$. Observe that this cannot be guaranteed by invoking neither continuity nor semi-continuity of the payoffs. As we showed in Example 1, both conditions are violated in the hybrid election game.

Let $(A, \mathcal{B}(A), \mu)$ be a Borel probability space, where $\mathcal{B}(A)$ is a Borel $\sigma$-algebra on $A$ and $\mu$ : $\mathcal{B}(A) \rightarrow \mathbb{R}_{+}$a probability measure on $\mathcal{B}(A)$. A real valued function $z: A \rightarrow \mathbb{R}$ is measurable with respect to $\mathcal{B}(A)$ if, for all $\alpha \in \mathbb{R},\{x \in A: z(x)<\alpha\} \in \mathcal{B}(A)$.

Lemma 7 For each $i=1,2, \pi_{i}: A \rightarrow \mathbb{R}$ is Borel measurable on $A$.

Proof. We prove the lemma for $\pi_{1}$. The argument for $\pi_{2}$ is identical. For all $\left(x_{1}, x_{2}\right) \in A$, let $\pi_{1}^{*}\left(x_{1}, x_{2}\right)=\left[1-H\left(1 / 2-F\left(S\left(x_{1}, x_{2}\right)\right)\right]\left(\psi\left(x_{1}, x_{2} ; \theta^{1}\right)+\chi_{1}\right)\right.$. By continuity, $\pi_{1}^{*}$ is measurable on $A$. Let $\bar{D}(A)=\left\{\left(x_{1}, x_{2}\right) \in A: x_{1}=x_{2} \neq \theta_{m}\right\}$. Clearly, $\left\{\left(x_{1}, x_{2}\right) \in A: \pi_{1}\left(x_{1}, x_{2}\right) \neq \pi_{1}^{*}\left(x_{1}, x_{2}\right)\right\}=$ $\bar{D}(A)$. Hence, since $\mu(\bar{D}(A))=0, \pi_{1}$ and $\pi_{1}^{*}$ are equivalent with respect to $\mu$; and, therefore, $\pi_{1}$ is Borel measurable on $A$, because a function equivalent to a measurable function is itself measurable (Kolmogorov and Fomin [19], pp. 289).

Now we complete the description of the mixed extension, extending parties' payoffs to the domain of mixed strategies. That is, for each $i=1,2$, we define the expected payoff on $\Delta(A)$ as $U_{i}\left(\mu_{1}, \mu_{2}\right)=\int_{A} \pi_{i}(x) d\left(\mu_{1}, \mu_{2}\right)$, where $\forall i, \mu_{i} \in \Delta\left(A_{i}\right)$. Observe that $U_{i}: \Delta(A) \rightarrow \mathbb{R}$ is well defined, because any Borel measurable function on $A$ is measurable in the associated product measure space. $^{24}$

Definition 5 A mixed strategy Nash equilibrium of $\mathcal{G}=\left[\left(A_{i}, \pi_{i}\right) ; i=1,2\right]$ is a pair of probability measures $\left(\mu_{1}^{*}, \mu_{2}^{*}\right) \in \Delta(A)$ such that, for all $\left(\mu_{1}, \mu_{2}\right) \in \Delta(A), U_{1}\left(\mu_{1}^{*}, \mu_{2}^{*}\right) \geq U_{1}\left(\mu_{1}, \mu_{2}^{*}\right)$ and $U_{2}\left(\mu_{1}^{*}, \mu_{2}^{*}\right) \geq U_{2}\left(\mu_{1}^{*}, \mu_{2}\right)$.

\footnotetext{
${ }^{24}$ Recall that $\pi_{i}(\cdot)$ is bounded, because $p(\cdot)$ and $\psi\left(\cdot ; \theta^{i}\right)$ are bounded on $A$. Therefore, by Lemma $7, \pi_{i}(\cdot)$ is $\mu$-integrable and its expected value is well defined.
} 
That is, a mixed strategy Nash equilibrium of $\mathcal{G}$ is a Nash equilibrium of the mixed extension $\overline{\mathcal{G}}=\left[\left(\Delta\left(A_{i}\right), U_{i}\right) ; i=1,2\right]$. We denote $M E(\mathcal{G})$ the set of all such equilibria. To prove that $M E(\mathcal{G})$ is nonempty is not a trivial matter, because $\mathcal{G}$ is an infinite action game with discontinuous payoffs. To solve this problem, we use Reny [24], according to which a (compact and Hausdorff) game possesses a mixed strategy Nash equilibrium if its mixed extension satisfies a condition called better reply security. This condition is defined as follows. Let $U: \Delta(A) \rightarrow \mathbb{R}^{2}$ be the mixed extension's vector payoff function, so that for all $\mu \in \Delta(A), U(\mu)=\left(U_{1}(\mu), U_{2}(\mu)\right):{ }^{25}$

Definition $6 \overline{\mathcal{G}}=\left[\left(\Delta\left(A_{i}\right), U_{i}\right) ; i=1,2\right]$ is better reply secure if for any $\left(\mu^{*}, U^{*}\right) \in \operatorname{cl}(\operatorname{gr}(U))$ such that $\mu^{*} \notin M E(\mathcal{G})$, there exists a party, say $i$, who can secure a payoff strictly above $U_{i}^{*}$ at $\mu^{*}{ }^{26}$

Proposition $3 \overline{\mathcal{G}}$ is better reply secure.

Proof. Assume, by contradiction, that there exists $\left(\mu^{*}, U^{*}\right) \in \operatorname{cl}(\operatorname{gr}(U))$ such that $\mu^{*} \notin M E(\mathcal{G})$ and no party $i=1,2$ can secure a payoff strictly above $U_{i}^{*}$ at $\mu^{*}$. This is equivalent to say that, for all $i$, all $\mu_{i} \in \Delta\left(A_{i}\right)$, and all $\delta>0, \exists \mu_{-i}(\delta)$ such that $\left\|\mu_{-i}(\delta)-\mu_{-i}^{*}\right\|<\delta$ and $U_{i}\left(\mu_{i}, \mu_{-i}(\delta)\right) \leq U_{i}^{*}$, where $\|\cdot\|$ denotes the norm in $\Delta(A)$. We disprove this claim as follows:

Step 1. There is a party, say 1 , and a strategy $\hat{\mu}_{1} \in \Delta\left(A_{1}\right)$, such that $U_{1}\left(\hat{\mu}_{1}, \mu_{2}^{*}\right)>U_{1}\left(\mu_{1}^{*}, \mu_{2}^{*}\right)$;

Step 2. There exists a proposal $\bar{x}_{1} \in A_{1}$ either, in the support of $\hat{\mu}_{1}$, or "close enough" to a platform in $\operatorname{supp}\left(\hat{\mu}_{1}\right)$, such that $\bar{x}_{1}$ is not an atom of $\mu_{2}^{*}$ and $U_{1}\left(\bar{x}_{1}, \mu_{2}^{*}\right)>U_{1}\left(\mu_{1}^{*}, \mu_{2}^{*}\right)$;

Step 3. By continuity of $U_{1}\left(\bar{x}_{1}, \cdot\right)$ in $\mu_{2}$ at $\mu_{2}^{*}$, party 1 can secure a payoff $U_{1}\left(\bar{x}_{1}, \mu_{2}^{*}\right)$ strictly greater than $U_{1}\left(\mu_{1}^{*}, \mu_{2}^{*}\right)$;

Step 4. Repeating the argument behind Step 2 and 3, party 2 can secure a payoff $U_{2}\left(\mu_{1}^{*}, \bar{x}_{2}\right)$ greater than or equal to $U_{2}\left(\mu_{1}^{*}, \mu_{2}^{*}\right)$;

Step 5. By Steps 3 and $4, U_{1}^{*}+U_{2}^{*}>U_{1}\left(\mu_{1}^{*}, \mu_{2}^{*}\right)+U_{2}\left(\mu_{1}^{*}, \mu_{2}^{*}\right)$;

Step 6. Finally, despite of the discontinuities of $\Pi=\pi_{1}+\pi_{2}$ on $D(A)$, for every sequence $\left\{\mu^{n}\right\} \subset \Delta(A)$ that converges to $\mu^{*}, \lim _{\mu^{n} \rightarrow \mu^{*}} \int_{A} \Pi(x) d \mu^{n}$ converges to $\int_{A} \Pi(x) d \mu^{*}$, which stands in contradiction with Step 5.

Now we offer more details for each of the previous steps. As it will become immediately clear, the crucial one is Step 2. Together with Step 3, it roughly says that party 1's profitable deviation from $\left(\mu_{1}^{*}, \mu_{2}^{*}\right)$ cannot be undercut by party 2 , by displacing a slightly amount of probability mass. The structure of the game ensures that party 1 can always protect his deviation payoff from this undermining behavior.

Step 1. Trivial. Since the strategy profile $\mu^{*}$ is not a mixed strategy equilibrium, without loss of generality we can assume that $\exists \hat{\mu}_{1} \in \Delta\left(A_{1}\right)$ such that

$$
U_{1}\left(\hat{\mu}_{1}, \mu_{2}^{*}\right)>U_{1}\left(\mu_{1}^{*}, \mu_{2}^{*}\right) .
$$

Step 2. Consider a platform $\hat{x}_{1} \in \operatorname{supp}\left(\hat{\mu}_{1}\right)$ such that $U_{1}\left(\hat{x}_{1}, \mu_{2}^{*}\right)>U_{1}\left(\mu_{1}^{*}, \mu_{2}^{*}\right)$. That proposal always exists. On the contrary, suppose that $\forall x_{1} \in \operatorname{supp}\left(\hat{\mu}_{1}\right), U_{1}\left(x_{1}, \mu_{2}^{*}\right) \leq U_{1}\left(\mu_{1}^{*}, \mu_{2}^{*}\right)$. Integrating

\footnotetext{
${ }^{25}$ The graph of $U$ and its closure are defined as before.

${ }^{26}$ As before, party $i$ can secure a payoff $\alpha \in \mathbb{R}$ at $\mu \in \Delta(A)$ if there exists $\bar{\mu}_{i} \in \Delta\left(A_{i}\right)$ such that, for all $\mu_{-i}^{\prime}$ in some open neighborhood of $\mu_{-i}, U_{i}\left(\bar{\mu}_{i}, \mu_{-i}^{\prime}\right) \geq \alpha$.
} 
with respect to $\hat{\mu}_{1}$, we have that $\int_{A_{1}} U_{1}\left(x_{1}, \mu_{2}^{*}\right) d \hat{\mu}_{1}=U_{1}\left(\hat{\mu}_{1}, \mu_{2}^{*}\right) \leq U_{1}\left(\mu_{1}^{*}, \mu_{2}^{*}\right)=\int_{A_{1}} U_{1}\left(\mu_{1}^{*}, \mu_{2}^{*}\right) d \hat{\mu}_{1}$, which contradicts (9). Therefore, if $\hat{x}_{1} \notin \mathcal{A}\left(\mu_{2}^{*}\right)$, we are done. Otherwise, we proceed as follows.

Without loss of generality, suppose $\theta^{1}<\theta_{m}$. Consider a platform $\bar{x}_{1} \in A_{1}$ close enough to $\hat{x}_{1}$, such that $\bar{x}_{1} \notin \mathcal{A}\left(\mu_{2}^{*}\right)$ and

$$
U_{1}\left(\bar{x}_{1}, \mu_{2}^{*}\right) \geq U_{1}\left(\hat{x}_{1}, \mu_{2}^{*}\right) .
$$

Since $\mu_{2}^{*}$ is a probability measure and it has at most countably many atoms, we show next that such a $\bar{x}_{1}$ always exists. To fix the notation, let $\hat{x}_{2}=\hat{x}_{1}$. By the argument behind Lemma 2 , for $\delta>0$ sufficiently small, there exists $\bar{x}_{1}=\hat{x}_{1}+\delta$ such that $\forall x_{2}^{\prime} \in B_{\delta}\left(\hat{x}_{2}\right), \pi_{1}\left(\bar{x}_{1}, x_{2}^{\prime}\right)>\pi_{1}\left(\hat{x}_{1}, \hat{x}_{2}\right)$. Integrating with respect to $\mu_{2}^{*}$ over $B_{\delta}\left(\hat{x}_{2}\right)$, we have $\int_{B_{\delta}\left(\hat{x}_{2}\right)} \pi_{1}\left(\bar{x}_{1}, x_{2}\right) d \mu_{2}^{*}>\frac{\chi_{1}}{2} \int_{B_{\delta}\left(\hat{x}_{2}\right)} d \mu_{2}^{*}$. Moreover, since $\frac{\chi_{1}}{2} \int_{B_{\delta}\left(\hat{x}_{2}\right)} d \mu_{2}^{*} \geq \frac{\chi_{1}}{2} \mu_{2}^{*}\left(\hat{x}_{2}\right)$, it follows that

$$
\int_{B_{\delta}\left(\hat{x}_{2}\right)} \pi_{1}\left(\bar{x}_{1}, x_{2}\right) d \mu_{2}^{*}>\frac{\chi_{1}}{2} \mu_{2}^{*}\left(\hat{x}_{2}\right) .
$$

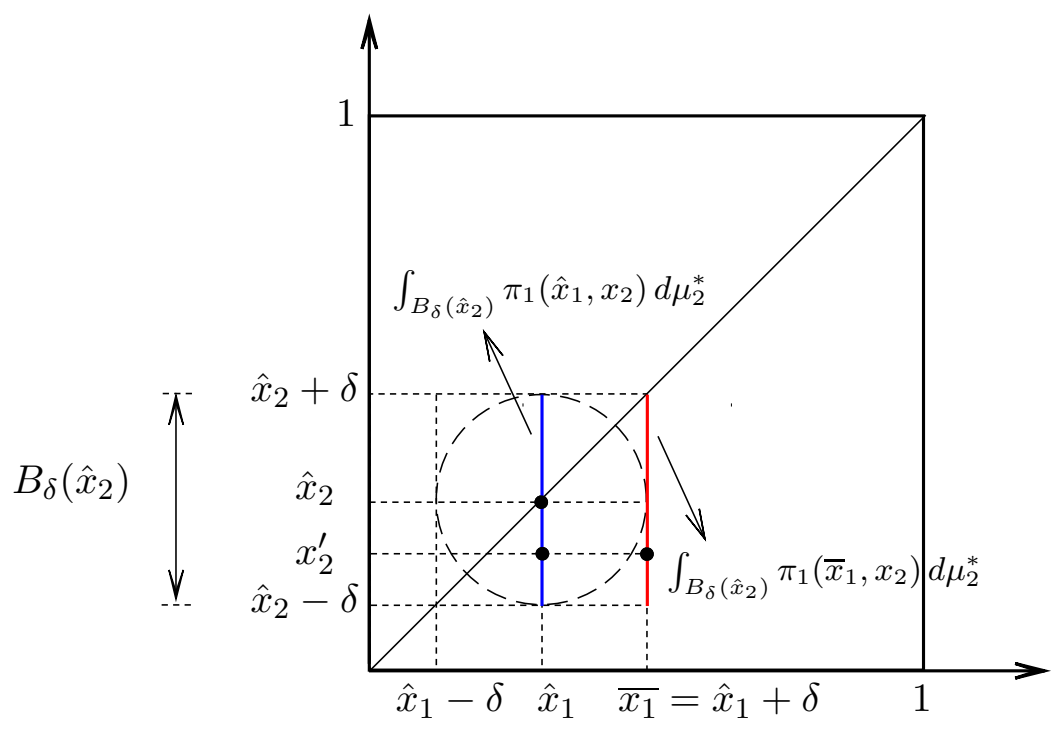

Figure 3: What's going on over $B_{\delta}\left(\hat{x}_{2}\right)$ ?

Note that

$$
\begin{gathered}
\int_{B_{\delta}\left(\hat{x}_{2}\right)} \pi_{1}\left(\hat{x}_{1}, x_{2}\right) d \mu_{2}^{*}=\frac{1}{2} \chi_{1} \mu_{2}^{*}\left(\hat{x}_{2}\right)+\int_{\hat{x}_{2}-\delta}^{\hat{x}_{2}}\left[1-H\left(\frac{1}{2}-F\left(1-\frac{x_{2}+\hat{x}_{1}}{2}\right)\right)\right] \cdot \\
\cdot\left(\psi\left(\hat{x}_{1}, x_{2} ; \theta^{1}\right)+\chi_{1}\right) d \mu_{2}^{*}+\int_{\hat{x}_{2}}^{\hat{x}_{2}+\delta}\left[1-H\left(\frac{1}{2}-F\left(\frac{x_{2}+\hat{x}_{1}}{2}\right)\right)\right] \cdot\left(\psi\left(\hat{x}_{1}, x_{2} ; \theta^{1}\right)+\chi_{1}\right) d \mu_{2}^{*} .
\end{gathered}
$$

Therefore, as $\delta \rightarrow 0, \int_{B_{\delta}\left(\hat{x}_{2}\right)} \pi_{1}\left(\hat{x}_{1}, x_{2}\right) d \mu_{2}^{*} \rightarrow \frac{1}{2} \chi_{1} \mu_{2}^{*}\left(\hat{x}_{2}\right)$, because the last two terms in the right hand side of (11) are areas behind two continuous functions, computed over an interval that becomes arbitrarily small as $\delta \rightarrow 0$ (see Figure 3 above). Thus, for $\delta$ small enough, we have that 


$$
\int_{B_{\delta}\left(\hat{x}_{2}\right)} \pi_{1}\left(\bar{x}_{1}, x_{2}\right) d \mu_{2}^{*} \geq \int_{B_{\delta}\left(\hat{x}_{2}\right)} \pi_{1}\left(\hat{x}_{1}, x_{2}\right) d \mu_{2}^{*}
$$

Let's see now what happens with these integrals over $B_{\delta}^{c}\left(\hat{x}_{2}\right)=A_{2}-B_{\delta}\left(\hat{x}_{2}\right)$. In order to guarantee that (10) holds, we want to prove that

$$
\int_{B_{\delta}^{c}\left(\hat{x}_{2}\right)} \pi_{1}\left(\bar{x}_{1}, x_{2}\right) d \mu_{2}^{*} \geq \int_{B_{\delta}^{c}\left(\hat{x}_{2}\right)} \pi_{1}\left(\hat{x}_{1}, x_{2}\right) d \mu_{2}^{*}
$$

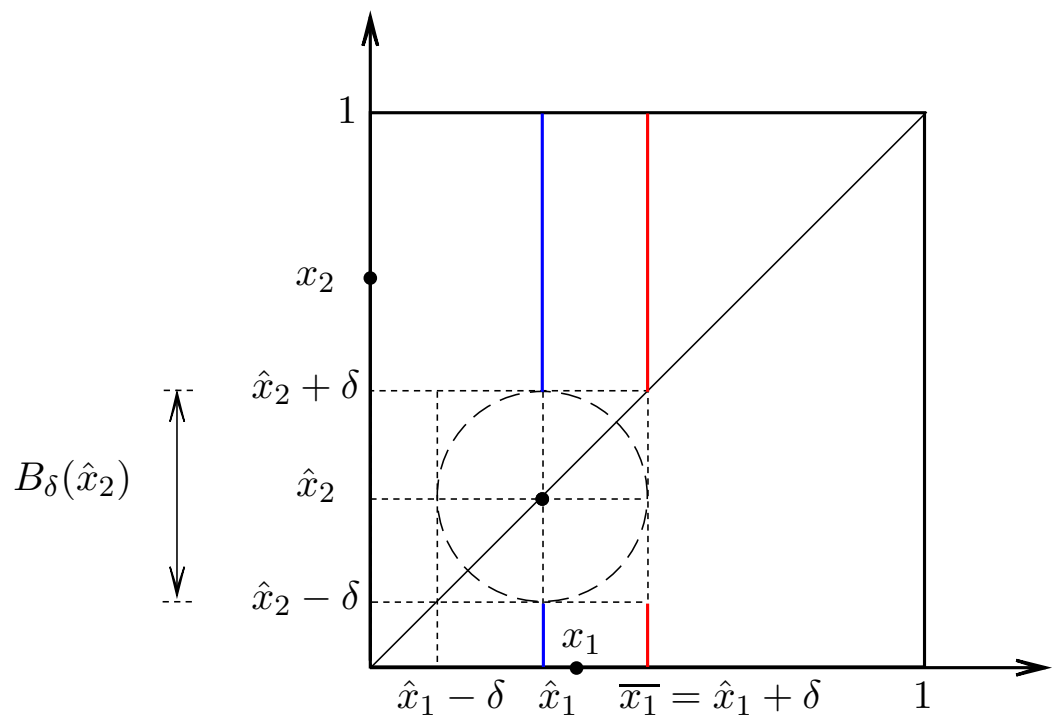

Figure 4: What's going on over $B_{\delta}^{c}\left(\hat{x}_{2}\right) ?$

The argument behind (13) is as follows. First, note that for every $x_{2} \in B_{\delta}^{c}\left(\hat{x}_{2}\right), \pi_{1}\left(\cdot, x_{2}\right)$ is continuous in $x_{1}$ at $\hat{x}_{1}$. In other words, given a $x_{2} \in B_{\delta}^{c}\left(\hat{x}_{2}\right), \forall \epsilon>0, \exists \lambda_{\epsilon}\left(x_{2}\right)>0$ such that, $\forall x_{1} \in B_{\lambda_{\epsilon}\left(x_{2}\right)}\left(\hat{x}_{1}\right), \pi_{1}\left(x_{1}, x_{2}\right)>\pi_{1}\left(\hat{x}_{1}, x_{2}\right)-\epsilon$. Fix $\epsilon>0$ and let $\underline{\lambda}_{\epsilon}=\inf \left\{\lambda_{\epsilon}\left(x_{2}\right): x_{2} \in B_{\delta}^{c}\left(\hat{x}_{2}\right)\right\}$. Then, for all $x_{1} \in B_{\underline{\lambda}_{\epsilon}}\left(\hat{x}_{1}\right)$, and all $x_{2} \in B_{\delta}^{c}\left(\hat{x}_{2}\right), \pi_{1}\left(x_{1}, x_{2}\right)>\pi_{1}\left(\hat{x}_{1}, x_{2}\right)-\epsilon$. Taking any $x_{1} \in$ $B_{\underline{\lambda}_{\epsilon}}\left(\hat{x}_{1}\right)$ and integrating with respect to $\mu_{2}^{*}$ over $B_{\delta}^{c}\left(\hat{x}_{2}\right)$, we have that $\int_{B_{\delta}^{c}\left(\hat{x}_{2}\right)} \pi_{1}\left(x_{1}, x_{2}\right) d \mu_{2}^{*}>$ $\int_{B_{\delta}^{c}\left(\hat{x}_{2}\right)}\left(\pi_{1}\left(\hat{x}_{1}, x_{2}\right)-\epsilon\right) d \mu_{2}^{*}$. However, for $\epsilon$ sufficiently small, $\int_{B_{\delta}^{c}\left(\hat{x}_{2}\right)} \epsilon d \mu_{2}^{*}$ is negligible. Hence,

$$
\int_{B_{\delta}^{c}\left(\hat{x}_{2}\right)} \pi_{1}\left(x_{1}, x_{2}\right) d \mu_{2}^{*} \geq \int_{B_{\delta}^{c}\left(\hat{x}_{2}\right)} \pi_{1}\left(\hat{x}_{1}, x_{2}\right) d \mu_{2}^{*} .
$$

If $\delta \leq \underline{\lambda}_{\epsilon}$, we are done, because (14) holds for any $x_{1} \in B_{\underline{\lambda}_{\epsilon}}\left(\hat{x}_{1}\right)$. In particular, for $\bar{x}_{1}=\hat{x}_{1}+\delta .^{27}$ Therefore, (12) and (13) would imply (10). Otherwise, if $\delta>\underline{\lambda}_{\epsilon}$, then we take as we illustrate in Figure 5 a proposal $x_{1}^{\prime}$ in the interval $\left(\hat{x}_{1}, \hat{x}_{1}+\underline{\lambda}_{\epsilon}\right)$, with the proviso that $x_{1}^{\prime} \notin \mathcal{A}\left(\mu_{2}^{*}\right)$. Again, since $\mu_{2}^{*}$ has at most countably many atoms, this alternative exists. Let $\delta^{\prime}=d\left(x_{1}^{\prime}, \hat{x}_{1}\right)$. Using the same argument behind (12), it follows that

\footnotetext{
${ }^{27}$ Recall that $\hat{x}_{2}+\delta \in B_{\delta}^{c}\left(\hat{x}_{2}\right)$, so that $\pi_{1}\left(\bar{x}_{1}, \cdot\right)$ may jump at $\hat{x}_{2}+\delta$. However, $\bar{x}_{1} \notin \mathcal{A}\left(\mu_{2}^{*}\right)$; i.e., $\mu_{2}^{*}$ assigns zero probability mass over $\hat{x}_{2}+\delta$. Thus, the (potential) discontinuity of $\pi_{1}\left(\bar{x}_{1}, \cdot\right)$ at $\hat{x}_{2}+\delta$ is irrelevant for the analysis.
} 


$$
\int_{B_{\delta^{\prime}}\left(\hat{x}_{2}\right)} \pi_{1}\left(x_{1}^{\prime}, x_{2}\right) d \mu_{2}^{*} \geq \int_{B_{\delta^{\prime}}\left(\hat{x}_{2}\right)} \pi_{1}\left(\hat{x}_{1}, x_{2}\right) d \mu_{2}^{*}
$$

Moreover, by (14),

$$
\int_{B_{\delta}^{c}\left(\hat{x}_{2}\right)} \pi_{1}\left(x_{1}^{\prime}, x_{2}\right) d \mu_{2}^{*} \geq \int_{B_{\delta}^{c}\left(\hat{x}_{2}\right)} \pi_{1}\left(\hat{x}_{1}, x_{2}\right) d \mu_{2}^{*}
$$

Thus, it remains to see what happens in the region $\Gamma=\left(\hat{x}_{2}-\delta, \hat{x}_{2}-\delta^{\prime}\right] \cup\left[\hat{x}_{2}+\delta^{\prime}, \hat{x}_{2}+\delta\right)$. Notice that $\int_{\Gamma} \pi_{1}\left(x_{1}^{\prime}, x_{2}\right) d \mu_{2}^{*}=\int_{\hat{x}_{2}-\delta}^{\hat{x}_{2}-\delta^{\prime}} \pi_{1}\left(x_{1}^{\prime}, x_{2}\right) d \mu_{2}^{*}+\int_{\hat{x}_{2}+\delta^{\prime}}^{\hat{x}_{2}+\delta} \pi_{1}\left(x_{1}^{\prime}, x_{2}\right) d \mu_{2}^{*}+\frac{\chi_{1}}{2} \mu_{2}^{*}\left(\hat{x}_{2}+\delta^{\prime}\right)$.

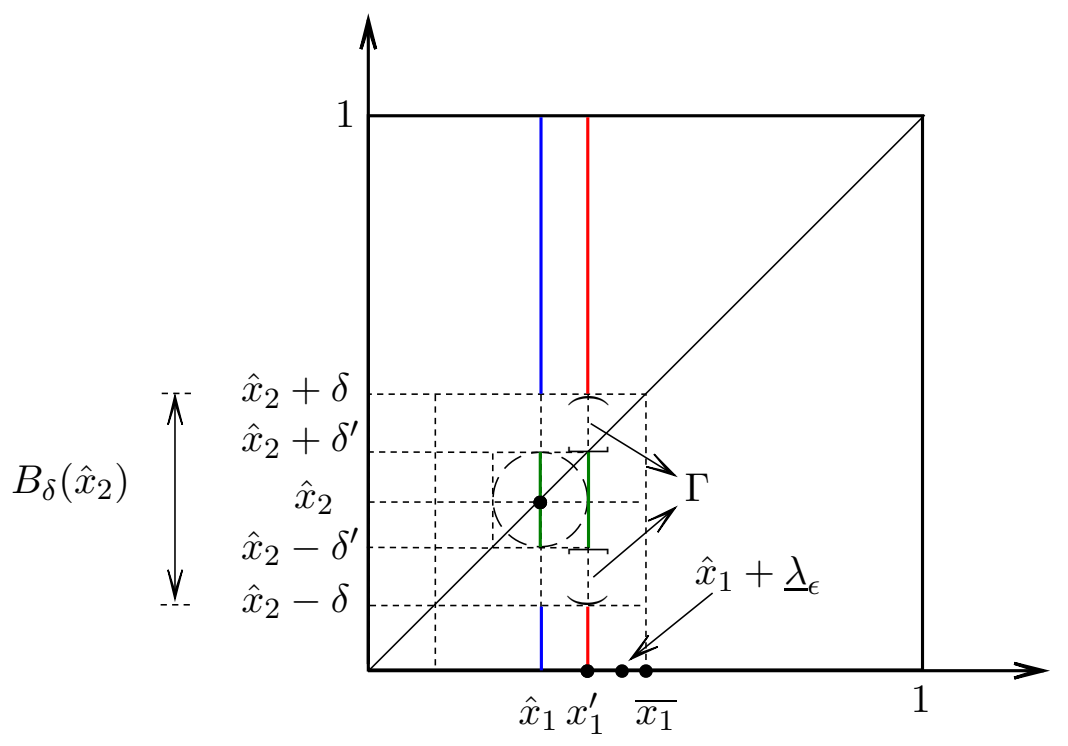

Figure 5: What's going on over $\Gamma$ ?

However, as $\delta \rightarrow 0, \delta$ and $\delta^{\prime}$ become arbitrarily close to each other. Therefore, since $x_{1}^{\prime} \notin$ $\mathcal{A}\left(\mu_{2}^{*}\right)$ (i.e. $\left.\mu_{2}^{*}\left(\hat{x}_{2}+\delta^{\prime}\right)=0\right)$ and $\pi_{1}\left(x_{1}^{\prime}, \cdot\right)$ is continuous in $x_{2}$ over $\left(\hat{x}_{2}-\delta, \hat{x}_{2}-\delta^{\prime}\right]$ and over $\left(\hat{x}_{2}+\delta^{\prime}, \hat{x}_{2}+\delta\right)$, respectively, the area behind $\pi_{1}\left(x_{1}^{\prime}, x_{2}\right)$ on $\Gamma$ is negligible. A similar argument implies that $\int_{\Gamma} \pi_{1}\left(\hat{x}_{1}, x_{2}\right) d \mu_{2}^{*}$ can also be ignored. Therefore, for $\delta$ sufficiently small, (15) and (16) imply that $U_{1}\left(x_{1}^{\prime}, \mu_{2}^{*}\right) \geq U_{1}\left(\hat{x}_{1}, \mu_{2}^{*}\right)$.

In short, by proposing $\hat{x}_{1}$ itself or, if $\hat{x}_{1} \in \mathcal{A}\left(\mu_{2}^{*}\right)$, by moving closer to $\hat{x}_{1}$ and choosing either $\bar{x}_{1}$ or $x_{1}^{\prime}$, the previous analysis shows that party 1 has a platform in his strategy set that it is not an atom of $\mu_{2}^{*}$, and that offers him an expected payoff strictly greater than $U_{1}\left(\mu_{1}^{*}, \mu_{2}^{*}\right)$. Next we prove that this payoff can also be secured.

Step 3. To simplify the exposition, let us generically refer to the platform of Step 2 as $\bar{x}_{1}$, understanding that this is the proposal which offers to party 1 a payoff strictly above $U_{1}\left(\mu_{1}^{*}, \mu_{2}^{*}\right)$ and $\bar{x}_{1} \notin \mathcal{A}\left(\mu_{2}^{*}\right)$. Then, $U_{1}\left(\bar{x}_{1}, \cdot\right)$ is continuous in $\mu_{2}$ at $\mu_{2}^{*}$. That is, for all $\epsilon>0$, there exists $\delta_{\epsilon}>0$ such that $U_{1}\left(\bar{x}_{1}, \tilde{\mu}_{2}\right)>U_{1}\left(\bar{x}_{1}, \mu_{2}^{*}\right)-\epsilon$ for all $\tilde{\mu}_{2}$ such that $\left\|\tilde{\mu}_{2}-\mu_{2}^{*}\right\|<\delta_{\epsilon}$. Therefore, for $\epsilon$ small enough, party 1 can secure $U_{1}\left(\bar{x}_{1}, \mu_{2}^{*}\right)$. That is, there is a strategy, $\bar{x}_{1}$, and $\delta>0$, such that $U_{1}\left(\bar{x}_{1}, \tilde{\mu}_{2}\right) \geq U_{1}\left(\bar{x}_{1}, \mu_{2}^{*}\right)$ for all $\tilde{\mu}_{2}$ such that $\left\|\tilde{\mu}_{2}-\mu_{2}^{*}\right\|<\delta$. 
Step 4. Consider a platform $x_{2} \in \operatorname{supp}\left(\mu_{2}^{*}\right)$ such that $U_{2}\left(\mu_{1}^{*}, x_{2}\right) \geq U_{2}\left(\mu_{1}^{*}, \mu_{2}^{*}\right)$. Clearly, $x_{2}$ always exists. Moreover, using the same argument behind Step 2, it follows that either $x_{2} \notin \mathcal{A}\left(\mu_{1}^{*}\right)$ or, if $x_{2} \in \mathcal{A}\left(\mu_{1}^{*}\right)$, then there exists a proposal $\bar{x}_{2} \in A_{2}$ close enough to $x_{2}$ such that $U_{2}\left(\mu_{1}^{*}, \bar{x}_{2}\right) \geq$ $U_{2}\left(\mu_{1}^{*}, x_{2}\right)$ and $\bar{x}_{2} \notin \mathcal{A}\left(\mu_{1}^{*}\right)$. Hence, invoking continuity of $U_{2}\left(\cdot, \bar{x}_{2}\right)$ in $\mu_{1}$ at $\mu_{1}^{*}$, (as we did in Step 3 for party 1$)$, we have that party 2 can secure a payoff $U_{2}\left(\mu_{1}^{*}, \bar{x}_{2}\right)$ greater than or equal to $U_{2}\left(\mu_{1}^{*}, \mu_{2}^{*}\right)$.

Step 5. By Step 3, $U_{1}\left(\mu_{1}^{*}, \mu_{2}^{*}\right)<U_{1}^{*}$. Otherwise, if $U_{1}^{*} \leq U_{1}\left(\mu_{1}^{*}, \mu_{2}^{*}\right)$, then party 1 would be able to secure a payoff strictly greater than $U_{1}^{*}$, contradicting the initial hypothesis. Similarly, by Step $4, U_{2}\left(\mu_{1}^{*}, \mu_{2}^{*}\right) \leq U_{2}^{*}$. Hence, $U_{1}^{*}+U_{2}^{*}>U_{1}\left(\mu_{1}^{*}, \mu_{2}^{*}\right)+U_{2}\left(\mu_{1}^{*}, \mu_{2}^{*}\right)$.

Step 6. Since we have assumed that $\left(\mu^{*}, U^{*}\right) \in \operatorname{cl}(\operatorname{gr}(U))$, there exists a sequence $\left\{\mu^{n}\right\} \subset \Delta(A)$ such that $\mu^{n} \rightarrow \mu^{*}$, and $\lim _{\mu^{n} \rightarrow \mu^{*}}\left(\int_{A}\left(\pi_{1}\left(x_{1}, x_{2}\right)+\pi_{2}\left(x_{1}, x_{2}\right)\right) d \mu^{n}\right)=U_{1}^{*}+U_{2}^{*} .{ }^{28}$ Using $\Pi=\pi_{1}+\pi_{2}$ to simplify the notation, by Step 5 it follows that

$$
\lim _{\mu^{n} \rightarrow \mu^{*}}\left(\int_{A} \Pi\left(x_{1}, x_{2}\right) d \mu^{n}\right)>\int_{A} \Pi\left(x_{1}, x_{2}\right) d \mu^{*} .
$$

Note that (17) can be rewritten as

$$
\lim _{\mu^{n} \rightarrow \mu^{*}} \int_{A-D(A)} \Pi(x) d \mu^{n}-\int_{A-D(A)} \Pi(x) d \mu^{*}>\frac{\chi_{1}+\chi_{2}}{2}\left(\int_{D(A)} d \mu^{*}-\lim _{\mu^{n} \rightarrow \mu^{*}} \int_{D(A)} d \mu^{n}\right) .
$$

However, $\Pi$ is continuous on $A-D(A)$ and, for all $i=1,2, \chi_{i} \in \mathbb{R}_{+}$. Hence, by weak convergence, both the LHS and the RHS of (18) tends to zero, a contradiction. Therefore, $\overline{\mathcal{G}}$ is better reply secure.

Now, we present the main result of the paper:

Theorem 2 The two-party hybrid electoral competition game has a mixed strategy Nash equilibrium; i.e., $M E(\mathcal{G}) \neq \emptyset$.

Proof. Immediate from Proposition 3 and Corollary 5.2 in Reny [24].

Comparing our result in Theorem 2 with Ball [4], there are two main differences to point out. First, in our model the probability of winning the election is endogenously derived, from parties' uncertainty about voters' preferences, instead of being given by an exogenous function. This is important because the properties required to ensure the existence of a MSNE are not imposed on that function, but on more fundamental primitives of the model. In addition, by explicitly modeling the electoral uncertainty, it is also possible to get a better understanding of the election game, which allowed us for example to prove in Section 3.3 the existence and uniqueness of a PSNE when parties' motivations are homogeneous.

On the other hand, our existence analysis is based on Reny's better reply security. Instead, Ball [4] relies on Dasgupta and Maskin [7], (namely, on Theorem 5b). In this regard, it is worth noting that better reply security is virtually an ordinal property, (Reny [24], pp. 1034), in the sense that, if $g_{i}: \mathbb{R} \rightarrow \mathbb{R}$ is continuous and strictly increasing for every $i$, then $\left[\left(\Delta\left(A_{i}\right), U_{i}\right) ; i=1,2\right]$ is better reply secure if and only if $\left[\left(\Delta\left(A_{i}\right), g_{i} \circ U_{i}\right) ; i=1,2\right]$ is. So, the results in Proposition 3 and Theorem 2 hold for every continuous and strictly monotone transformation of parties' payoffs. In particular, they can be extended to the alternative specification of the MMA discussed in Section 2.

\footnotetext{
${ }^{28}$ Since $A_{i}$ is compact and $\pi_{i}$ is bounded, the sequence $\left\{\int_{A} \Pi(x) d \mu^{n}\right\}$ always converges when $\mu^{n} \rightarrow \mu^{*}$. Thus, without loss of generality, we assume that the $\operatorname{limit}, \lim _{\mu^{n} \rightarrow \mu^{*}}\left(\int_{A} \Pi(x) d \mu^{n}\right)$, always exists.
} 


\section{Final remarks}

This paper analyzes a traditional unidimensional, two-party electoral competition game with electoral uncertainty. The novelty is that, instead of assuming that parties have a single and homogeneous motive, as it is usually the case in the existent literature, we suppose that they may be distinctly concerned with both, winning the election, and the policy implemented after the contest. The main results obtained from this departure are as follows.

Firstly, in contrast with the standard election game with ideological parties, the payoff functions of the hybrid game are shown to be neither continuous nor semi-continuous on the strategy space (Example 1). Moreover, conditional payoffs might also violate quasi-concavity (Example 2). Thus, in spite of being payoff secure (Lemma 2) and reciprocally upper semi-continuous (Lemma 3), the hybrid election game does not always possess a pure strategy Nash equilibrium. In fact, its existence is guaranteed only if political parties have homogenous interest in power (Theorem 1), in which case the equilibrium is also unique (Proposition 2).

Secondly, to deal with the case of heterogeneous motives, we prove that the mixed extension of the hybrid electoral competition game satisfies better reply security for all parametric conditions (Proposition 3). Thus, using Reny [24], we show that a Nash equilibrium always exists (Theorem 2 ), though it may be one in mixed strategies. This result extends Ball [4] to the case where the probability of winning function is endogenously derived. And, together with Theorem 1, it generalizes previous existence results in unidimensional electoral competition.

Finally, the analysis of the uniform distribution case carried out in Section 3.2 also suggests that, when parties have mixed and probably heterogeneous interests in power, the interaction of four variables shape equilibrium outcomes. These variables are the electoral uncertainty, parties' ideology, the aggregate opportunism and its distribution across parties. Depending on the values of these parameters, we might have a PSNE or a MSNE. Moreover, Saporiti [28] has showed that we might end up with both parties proposing platforms at the median position (i.e., with full convergence); with each one playing in its own ideological arena (i.e., with policy divergence); or with both on the same side of the political spectrum. ${ }^{29}$

In particular, when the opportunism is large compared with the electoral uncertainty, and asymmetrically distributed, the support of each MSNE is a closed interval located on one side of the median voter's ideal point, and is the same for both candidates. Probability distributions are atomless in the interior of this set, and concentrate on the support's end points as uncertainty increases. Equilibria in mixed strategies vanish above a critical level of uncertainty, beyond which each party plays a pure strategy in its ideological side (Saporiti [28]).

Of course, these results stand in sharp contrast with the standard prediction of the Downsian game where, regardless of the level of electoral uncertainty, both parties locate at the median ideal policy. They substantially differ from Wittman equilibria too, because in that case parties' ideologies constitute the main driven force of electoral outcomes. It is left for a future work to explore the validity of these findings in more general models of electoral competition.

\footnotetext{
${ }^{29}$ Some of these predictions resemble Mitchell [21].
} 


\section{References}

[1] Aragones, E. and T. Palfrey, (2005), Electoral competition between two candidates of different quality: The effects of candidate ideology and private information, in: Austen-Smith, D and J. Duggan, (eds), Social Choice and Strategic Decisions, New York: Springer-Verlag, pp. 93-112.

[2] Aragones, E. and T. Palfrey, (2002), Mixed equilibrium in a Downsian model with a favorite candidate, Journal of Economic Theory 103, 131-61.

[3] Austen-Smith, D. and J. Banks, (2005), Positive Political Theory II: Strategy and Structure, Ann Arbor: The University of Michigan Press, pp. 253-332.

[4] Ball, R., (1999), Discontinuity and nonexistence of equilibrium in the probabilistic spatial voting model, Social Choice and Welfare 16, 533-55.

[5] Bernhardt, D., Duggan, J. and F. Squintani, (2007), Electoral competition with privately informed candidates, Games and Economic Behavior 58, 1-29.

[6] Calvert, R., (1985), Robustness of the multidimensional voting model: Candidate motivations, uncertainty, and convergence, American Journal of Political Science 29, 270-86.

[7] Dasgupta, P. and E. Maskin, (1986), The existence of equilibrium in discontinuous economic games, Review of Economic Studies 53, 1-26.

[8] Downs, A., (1957), An Economic Theory of Democracy, New York: Harper Collins.

[9] Duggan, J., (2004), Candidate objectives and electoral equilibrium, typescript.

[10] Duggan, J., (2005), Candidate objectives and electoral equilibrium, in: Weingast, B. and D. Wittman, (eds), The Oxford Handbook of Political Economy, Oxford: Oxford University Press, pp. 64-83.

[11] Duggan, J. and M. Jackson, (2005), Mixed strategy equilibrium and deep covering in multidimensional electoral competition, typescript.

[12] Duggan, J. and M. Fey, (2005), Electoral competition with policy-motivated candidates, Games and Economic Behavior 51, 490-522.

[13] Ferejohn, J., (1986), Incumbent performance and electoral control, Public Choice 50, 5-26.

[14] Groseclose, T, (2001), A model of candidate location when one candidate has a valence advantage, American Journal of Political Science 45, 862-86.

[15] Hinich, M., (1977), Equilibrium in spatial voting: The median voter result is an artifact, Journal of Economic Theory 16, 208-19.

[16] Hoernig, S., (2007), Bertrand games and sharing rules, Economic Theory 31, 573-85.

[17] Hotelling, H., (1929), Stability in competition, Economic Journal 39, 41-57.

[18] Kramer, G., (1978), Existence of electoral equilibrium, in: Ordeshook, P., (ed), Game Theory and Political Science, New York: New York University Press, pp. 223-28.

[19] Kolmogorov, A. and S. Fomin, (1975), Introductory Real Analysis, New York: Dover Publications, INC.

[20] Laslier, J., (2000), Interpretations of electoral mixed strategies, Social Choice and Welfare 17, 283-92.

[21] Mitchell, D., (1987), Candidate behavior under mixed motives, Social Choice and Welfare 4, 153-60. 
[22] Morton, R., (1993), Incomplete information and ideological explanations of platform divergence, Americal Political Science Review 87, 382-92.

[23] Ortuño Ortín, I., (2002), Ideological versus Downsian political competition, Social Choice and Welfare 19, 551-67.

[24] Reny, P., (1999), On the existence of pure and mixed strategy Nash equilibria in discontinuous games, Econometrica 67, 1029-56.

[25] Roemer, J., (1994), A theory of policy differentiation in single issue electoral politics, Social Choice and Welfare 11, 355-80.

[26] Roemer, J., (1997), Political-economic equilibrium when parties represent constituents, Social Choice and Welfare 14, 479-502.

[27] Roemer, J., (2001), Political Competition, Cambridge: Harvard University Press.

[28] Saporiti, A., (2007), Mixed motives and electoral competition: equilibrium analysis and experimental results, typescript.

[29] Vives, X., (1999), Oligopoly Pricing, Cambridge: MIT Press.

[30] Wittman, D., (1983), Candidate motivation: A synthesis of alternative theories, American Political Science Review 77, 142-57.

[31] Wittman, D., (1990), Spatial strategies when candidates have policy preferences, in: Enelow, J. and M. Hinich, (eds), Advances in the Spatial Theory of Voting, Cambridge: Cambridge University Press, pp. 66-98. 